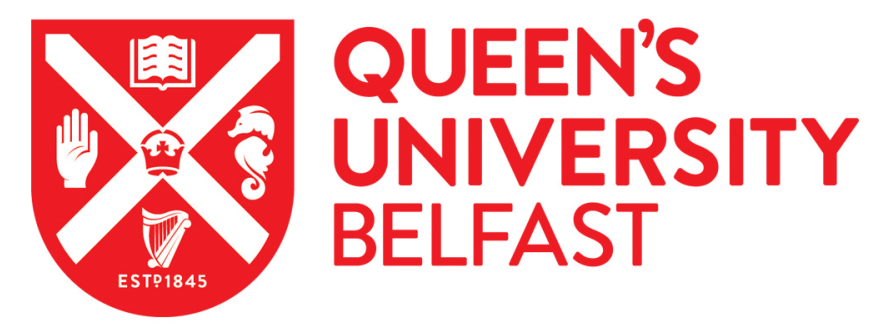

\title{
Doppler tomography as a tool for detecting exoplanet atmospheres
}

Watson, C. A., de Mooij, E. J. W., Steeghs, D., Marsh, T. R., Brogi, M., Gibson, N. P., \& Matthews, S. (2019). Doppler tomography as a tool for detecting exoplanet atmospheres. Monthly Notices of the Royal Astronomical Society, 490(2), 1991-2006. https://doi.org/10.1093/mnras/stz2679

Published in:

Monthly Notices of the Royal Astronomical Society

Document Version:

Publisher's PDF, also known as Version of record

Queen's University Belfast - Research Portal:

Link to publication record in Queen's University Belfast Research Portal

Publisher rights

Copyright 2019 Oxford University Press. This work is made available online in accordance with the publisher's policies. Please refer to any applicable terms of use of the publisher.

\section{General rights}

Copyright for the publications made accessible via the Queen's University Belfast Research Portal is retained by the author(s) and / or other copyright owners and it is a condition of accessing these publications that users recognise and abide by the legal requirements associated with these rights.

Take down policy

The Research Portal is Queen's institutional repository that provides access to Queen's research output. Every effort has been made to ensure that content in the Research Portal does not infringe any person's rights, or applicable UK laws. If you discover content in the Research Portal that you believe breaches copyright or violates any law, please contact openaccess@qub.ac.uk. 


\title{
Doppler tomography as a tool for detecting exoplanet atmospheres
}

\author{
C. A. Watson, ${ }^{1 \star}$ E. J. W. de Mooij ${ }^{\oplus},{ }^{1,2}$ D. Steeghs, ${ }^{3}$ T. R. Marsh, ${ }^{3}$ M. Brogi, ${ }^{3}$ \\ N. P. Gibson ${ }^{\oplus 1,4}$ and S. Matthews ${ }^{1}$ \\ ${ }^{1}$ Astrophysics Research Centre, Queen's University Belfast, Belfast BT7 1NN, UK \\ ${ }^{2}$ School of Physical Sciences and Centre for Astrophysics \& Relativity, Dublin City University, Glasnevin, Dublin 9, Ireland \\ ${ }^{3}$ Department of Physics, University of Warwick, Coventry CV4 7AL, UK \\ ${ }^{4}$ School of Physics, Trinity College Dublin, The University of Dublin, Dublin 2, Ireland
}

Accepted 2019 September 13. Received 2019 September 5; in original form 2019 April 5

\begin{abstract}
High-resolution Doppler spectroscopy is a powerful tool for identifying molecular species in the atmospheres of both transiting and non-transiting exoplanets. Currently, such data are analysed using cross-correlation techniques to detect the Doppler shifting signal from the orbiting planet. In this paper we demonstrate that, compared to cross-correlation methods currently used, the technique of Doppler tomography has improved sensitivity in detecting the subtle signatures expected from exoplanet atmospheres. This is partly due to the use of a regularizing statistic, which acts to suppress noise, coupled to the fact that all the data is fit simultaneously. In addition, we show that the technique can also effectively suppress contaminating spectral features that may arise due to overlapping lines, repeating line patterns, or the use of incorrect linelists. These issues can confuse conventional cross-correlation approaches, primarily due to aliasing issues inherent in such techniques, whereas Doppler tomography is less susceptible to such effects. In particular, Doppler tomography shows exceptional promise for simultaneously detecting multiple line species (e.g. isotopologues), even when there are high contrasts between such species - and far outperforms current crosscorrelation function (CCF) in this respect. Finally, we demonstrate that Doppler tomography is capable of recovering molecular signals from exoplanets using real data, by confirming the strong detection of $\mathrm{CO}$ in the atmosphere of $\tau$ Boo b. We recover a signal with a planetary radial velocity semi-amplitude $K_{\mathrm{p}}=109.6 \pm 2.2 \mathrm{~km} \mathrm{~s}^{-1}$, in excellent agreement with the previously reported value of $110.0 \pm 3.2 \mathrm{~km} \mathrm{~s}^{-1}$.
\end{abstract}

Key words: line: profiles - techniques: spectroscopic - planets and satellites: atmospheres planets and satellites: individual: $\tau$ Boo-b.

\section{INTRODUCTION}

Ground-based high-resolution Doppler spectroscopy has proven itself to be a powerful tool for probing the atmospheres of both transiting and non-transiting hot-Jupiters. Initial attempts to use time-series high-resolution Doppler spectroscopy to provide direct detections of hot-Jupiter atmospheres concentrated on trying to measure reflected starlight from the planets in the optical (e.g. Collier Cameron et al. 1999; Leigh et al. 2003a,b). Unfortunately, the low optical albedos of hot-Jupiters meant that these earlier attempts were unsuccessful. More recently, Martins et al. (2015) have claimed evidence for detected reflected starlight from $51 \mathrm{Peg}$

^E-mail: c.a.watson@qub.ac.uk b using Doppler spectroscopy, but this has since been challenged by Hoeijmakers, Snellen \& van Terwisga (2018).

Snellen et al. (2010) were the first to successfully use highresolution Doppler spectroscopy to detect the atmosphere of an exoplanet. In this case they used the CRyogenic Infra-Red Echelle Spectrograph (CRIRES) Spectrograph on the Very Large Telescope (VLT) to detect CO absorption during the transit of HD209458b. This was followed by the detection of CO molecular absorption from the dayside of the non-transiting hot-Jupiter $\tau$ Boo b by both Brogi et al. (2012) and Rodler, Lopez-Morales \& Ribas (2012) (with water vapour later found for the same planet by Lockwood et al. 2014). Since these discoveries, additional detections (including for other molecular species such $\mathrm{H}_{2} \mathrm{O}$ and $\mathrm{TiO}$ ) have also been reported for 51 Peg b (Brogi et al. 2013; Birkby et al. 2017), HD189733b (Birkby et al. 2013; de Kok et al. 2013; Brogi et al. 2016; Brogi 
et al. 2018), HD179949b (Brogi et al. 2014), upsilon Andromedae b (Piskorz et al. 2017), HD88133b (Piskorz et al. 2016), and WASP33b (Nugroho et al. 2017).

In addition to probing exoplanet atmospheres, high-resolution Doppler spectroscopy also enables the radial velocity motion of the planet to be determined, effectively reducing the system to a double-lined spectroscopic binary. As a result, the absolute mass of the planet can be determined even for non-transiting planets. With new instrumentation such as CRIRES+ soon to come online, the capability of high-resolution spectroscopy to drive forward our understanding of the fundamental parameters and atmospheres of short-period exoplanets will greatly increase. For example, the wider wavelength range coverage of CRIRES $+(\sim 6$ times wider than that of CRIRES) will enable a far larger number of spectral lines from molecular species such as $\mathrm{H}_{2} \mathrm{O}$ and $\mathrm{CH}_{4}$ to be captured. Since the signal scales roughly as $\sqrt{n}$, where $n$ is the number of lines observed (different line strengths and signal-to-noise as a function of wavelength complicate this assumption), such instruments promise far greater sensitivity to atmospheric signatures in the near-future.

Despite this, the application of high-resolution Doppler spectroscopy to exoplanet atmospheres is still limited, and the analyses still revolve around the use of cross-correlation functions. In this paper, we demonstrate that the application of Doppler tomography has a number of clear advantages over such cross-correlation techniques. We begin by briefly describing the technique in Section 2 and in Section 3 we apply Doppler tomography to detect $\mathrm{CO}$ from $\tau$ Boo b using the data of Brogi et al. (2012). We then test the ability of Doppler tomography relative to more conventional crosscorrelation techniques in Section 4. We present our conclusions in Section 5. Finally, we look at further adaptations and potential for novel uses of Doppler tomography for exoplanet atmosphere studies in Section 6.

\section{DOPPLER TOMOGRAPHY}

The technique of Doppler tomography was developed by Marsh \& Horne (1988) and is normally used to study close-binary systems. As applied to binaries, the technique aims to recover a modelindependent map in velocity space that resolves the distribution of line emission and/or absorption within the binary using a time-series of spectra. In essence, Doppler tomography assumes that orbiting material (whether that be a parcel of material in an accretion disc, or emission from a planet) traces out a sinusoidal radial velocity curve of the form,

$v_{R}(\phi)=\gamma-v_{x} \cos 2 \pi \phi+v_{y} \sin 2 \pi \phi$.

Here, $\phi$ is the orbital phase, $\gamma$ is the systemic velocity of the system, and $v_{x}$ and $v_{y}$ are the radial velocity semi-amplitudes of the cosine and sine terms, respectively. One can then use the information encoded in line-profiles observed as a function of orbital phase to calculate the strength of the line emission/absorption as a function of velocity. This, in turn, can be used to construct a velocity 'image' or map of the orbiting material in velocity space, defined as the strength of the line emission/absorption as a function of velocity, $I\left(v_{x}, v_{y}\right)$.

Fig. 1 presents a simple schematic outlining this process. Here we have a 'real' signal with $\mathrm{v}_{x}=0 \mathrm{~km} \mathrm{~s}^{-1}$ and $\mathrm{v}_{y}=30 \mathrm{~km} \mathrm{~s}^{-1}$. Integrating the observed flux along the correct radial-velocity curve (given by the dashed line 'A' in Fig. 1) results in a strong signal at the corresponding pixel in the velocity map (right-hand panel of Fig. 1). Integrating the flux along a radial-velocity curve that is slightly offset from the true signal (e.g. path 'B') would produce

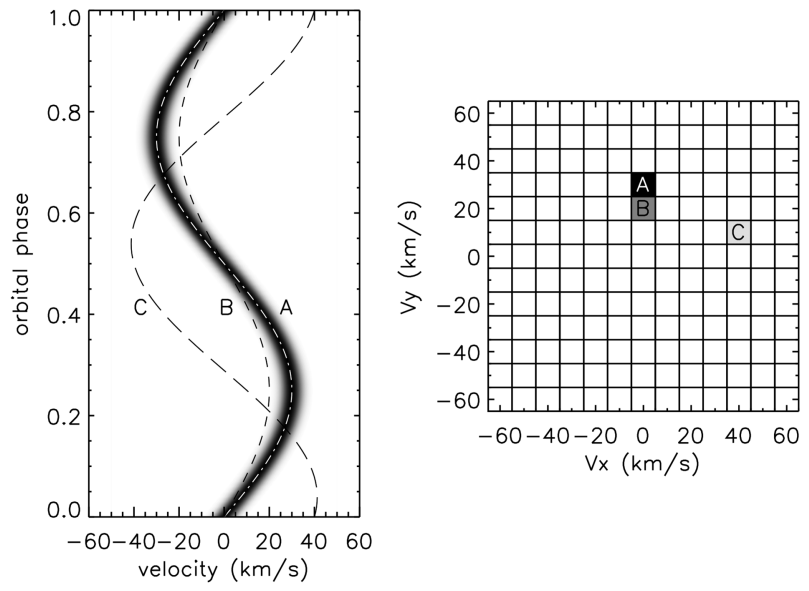

Figure 1. Left-hand panel: schematic showing an observed signal following a radial-velocity path described by equation (1) with $v_{x}=0 \mathrm{~km} \mathrm{~s}^{-1}$ and $v_{y}=30 \mathrm{~km} \mathrm{~s}^{-1}$. The dot-dashed line, 'A', and dashed lines ' $\mathrm{B}$ ' and ' $\mathrm{C}$ ' show different radial-velocity paths - with path A matching the observed signal. The right-hand panel depicts a velocity, or Doppler, map where the flux in each pixel represents a different integral along a path in the data given by equation (1) and the respective $v_{x}$ and $v_{y}$ values. Where such a path is close to the true one, the result is a strong signal in the corresponding pixel (in this case pixel A).

a reduced signal, since there is a reduced overlap with the real signal. Finally, path ' $\mathrm{C}$ ' traces a radial-velocity path with minimal overlap with the real signal, resulting in little signal appearing in the corresponding pixel in the velocity map.

Strictly speaking, the above describes the process of backprojection, where each pixel in the velocity map represents the integral of the signal along each individual radial velocity path described by equation (1). However, unlike back-projection, Doppler tomography fits the observed data simultaneously across all the observed lines and over all observed phases subject to assumptions regarding the expected modulation of the amplitude of the signal. In its most basic form, Doppler tomography assumes that a point is equally visible at all phases, and thus attributing a signal to paths $\mathrm{B}$ and $\mathrm{C}$ in Fig. 1 would yield a poorer fit to the data than just assigning all the signal to path A. As a result, Doppler tomography returns a much weaker (or no) signal at incorrect velocities in the resultant Doppler map relative to standard back-projection techniques. For the same reason, aliasing effects common to backprojection approaches are also greatly reduced (see Section 4.2). We note that Doppler tomography is not just constrained to assuming that the signal strength is constant at all orbital phases, but that it can also be modified to deal with a modulating signal. In the context of exoplanet atmospheres, this means Doppler tomography is able to deal with modulating signals that may arise from day-night phase variations, for example.

Although Doppler tomography has mainly been applied to cataclysmic variable binaries, primarily as a tool to probe the accretion regions in such systems, it has shown itself to be remarkably versatile - and its adaptation to a star-planet system is straight-forward. For this work, we assume that the planet is on a circular orbit, and thus the planetary radial-velocity variation can be described by equation (1) with $v_{x}=0 \mathrm{~km} \mathrm{~s}^{-1}$ and $v_{y}=K_{\mathrm{p}}$, where $K_{\mathrm{p}}$ is the radial-velocity semi-amplitude of the planet. The system can then be represented as an image in velocity space, which is the line emission/absorption strength as a function of velocity, $I\left(v_{x}, v_{y}\right)-$ as depicted in the right-hand panel of Fig. 1. If each pixel in velocity 
space has a width $\mathrm{d} v_{x}$ and $\mathrm{d} v_{y}$, then the contribution from that pixel is given by,

$I\left(v_{x}, v_{y}\right)\left[g\left(v-v_{R}\right)\right] \mathrm{d} v_{x} \mathrm{~d} v_{y}$,

where $g$ represents the line-profile shape from any point in the image and includes effects such as instrumental broadening as well as line broadening mechanisms.

The contribution of the lines from each position in our velocity map between $v$ and $v+\mathrm{d} v$ at orbital phase $\phi$ can then be determined by carrying out the integral,

$f(v, \phi)=\int_{-\infty}^{\infty} \int_{-\infty}^{\infty} I\left(v_{x}, v_{y}\right) g\left(v-v_{R}\right) \mathrm{d} v_{x} \mathrm{~d} v_{y}$,

where the integral limits are, in practice, set to cover an appropriate range in radial velocity.

The line profile at any orbital phase can be thought of as a projection of the velocity-space image along the direction determined by the orbital phase. In Doppler tomography, the reverse process is performed and a time-series of line profiles taken at different phases are inverted to construct a velocity image. From equation (1), different values of $v_{R}$ define a whole set of parallel straight lines across the Doppler map, with the direction dependent upon the orbital phase. For example, orbital phase 0 corresponds to a projection along the positive $\mathrm{v}_{y}$ axis, while phase 0.25 corresponds to a projection along the positive $\mathrm{v}_{x}$ axis. Thus the formation of the synthetic line profile for comparison to the data set at a particular phase can be thought of as a projection of the Doppler map along a direction defined by the orbital phase. Technically, all projections can be covered in half-an-orbit, since projections separated by halfan-orbit are merely mirror images of each other.

Broadly speaking, there are two primary techniques employed to perform the inversion: Fourier-filtered back-projection or the use of the maximum entropy method (MEM). We do not discuss the back-projection method here and refer the reader to numerous reviews on the topic (e.g. Marsh 2001), other than to note that it is limited in practice as it cannot take into account effects such as high line optical depths, blended lines, and any bad data need to be interpolated over. The MEM approach is more flexible and has greater tolerance to inconsistencies within the data set, and it is this version that we implement.

In the MEM reconstruction method, the intensities of the pixels in the velocity image are adjusted in order to fit the observed data to a target reduced $\chi^{2}$. The $\chi^{2}$ is not minimized, however, as this results in a map dominated by noise. Instead, the data are fit until the predicted and observed data are consistent. Since there are a number of different images that could satisfy this situation, a regularization statistic is implemented to select one, and the image of maximum entropy (the map with least information content relative to some comparison map - called the 'default map') is selected. The default map may encode prior information on the expected line distribution, or a smoothed (e.g. Gaussian smoothed) version of itself which helps preserve narrow features - but in its least informative form is simply a uniform map. Thus, Doppler tomography can be thought of as returning a velocity map of the system that contains the least amount of information required to describe the data to the required $\chi^{2}$.

The power of the MEM is that it requires no special provision to deal with missing data, it takes into account the error bars on the data, and the maximum entropy regularization statistic tends to suppress the growth of noise (which acts to increase the information content of the velocity map) in the final results. For more details on Doppler tomography, we refer the reader to the many extensive reviews of the technique undertaken in the past (e.g. Marsh 2001; Morales-Rueda 2004; Richards 2004; Schwope et al. 2004; Steeghs 2004; Vrtílek et al. 2004). We now turn to outlining its application to the high-resolution study of exoplanet atmospheres. In order to demonstrate the applicability of Doppler tomography to studying the Doppler shifted signatures of exoplanet atmospheres, we first apply it in the next Section to the exoplanet $\tau$ Boo b.

\section{DOPPLER TOMOGRAPHY OF $\tau$ Boob}

To demonstrate Doppler tomography's capability to recover the signal from molecules in real exoplanet data, we used CRIRES (Kaeufl, Ballester \& et al. 2004) data for $\tau$ Boo b from Brogi et al. (2012), who used it to successfully detect CO in the atmosphere of this non-transiting planet. This data set consists of 452 spectra obtained over three epochs amounting to a total of $\sim 18 \mathrm{~h}$ of observing time. We have implemented exactly the same data reduction and pre-processing steps as Brogi et al. (2012), including systematics removal and adoption of their orbital phases corrected from the original Butler et al. (2006) orbital solution, and we refer the reader to that paper for details.

For the Doppler tomography analysis we used a CO linelist for the ${ }^{12} \mathrm{C}^{16} \mathrm{O}$ isotopologue obtained from the HITRAN data base (Rothman et al. 2013) using HAPI (Kochanov et al. 2016). HAPI was also used to calculate the expected strengths of the lines for a temperature of $1700 \mathrm{~K}$ and a pressure of $0.1 \mathrm{bar}$. The results from this were subsequently fitted in IDL to generate a linelist file to use in the analysis, a total of 122 of the strongest CO lines in the wavelength range covered were used, with the strength of the faintest line at $\sim 2.5$ per cent that of the strongest line. This cut was made in order to retain a sufficient number of lines for use in Doppler tomography while removing the weakest lines that will have increasingly smaller contributions to the final reconstruction. A uniform default map was employed during the Doppler tomography reconstruction, and the velocity image consisted of 300 by 300 pixels with a $1.5 \mathrm{~km} \mathrm{~s}^{-1}$ stepsize. The same orbital phases as those in the Brogi et al. (2012) analysis were adopted, and the known systemic velocity of $\tau$ Boo was removed from the data. Hence any planetary signal should lie on the $v_{x}=0 \mathrm{~km} \mathrm{~s}^{-1}$ line in the Doppler tomogram.

We ran the Doppler tomogram to a reduced $\chi^{2}=0.96346$, and the resulting map can be found in Fig. 2. The CO signal from the planet can clearly be seen at the expected position with a planetary radial velocity semi-amplitude $\left(K_{\mathrm{p}}=v_{y}\right)$ of $109.6 \pm 2.2 \mathrm{~km} \mathrm{~s}^{-1}$. The uncertainty on $K_{\mathrm{p}}$ was determined by fitting a two-dimensional Gaussian to the planet signal in the Doppler map and adopting the $\sigma$-width of the Gaussian along the $\mathrm{v}_{y}$ axis. Our measured $K_{\mathrm{p}}$ is in excellent agreement with the value of $110.0 \pm 3.2 \mathrm{~km} \mathrm{~s}^{-1}$ reported by Brogi et al. (2012), and demonstrates that Doppler tomography can be used to directly detect exoplanet atmospheres.

We note that the fact we were able to fit to a reduced $\chi^{2}$ of less than 1 indicates that we have slightly overestimated the uncertainties on the data. In the case of weak signals (as expected in the study of exoplanet atmospheres) there is normally a relatively narrow $\chi^{2}$ window over which a satisfactory Doppler tomogram can be recovered. Fitting to higher aim $\chi^{2}$,s results in a blank featureless map, while conversely fitting to too low a $\chi^{2}$ results in a map dominated by noise. Thus, while the exact choice of aim $\chi^{2}$ is somewhat subjective, it is obvious when one lies outside a reasonable range. In the case of $\tau$ Boo $b$, if we fit to a reduced $\chi^{2}=1$ then, while the planet signal is still present, the Doppler map is poorly constrained by the data and does not converge. However, 


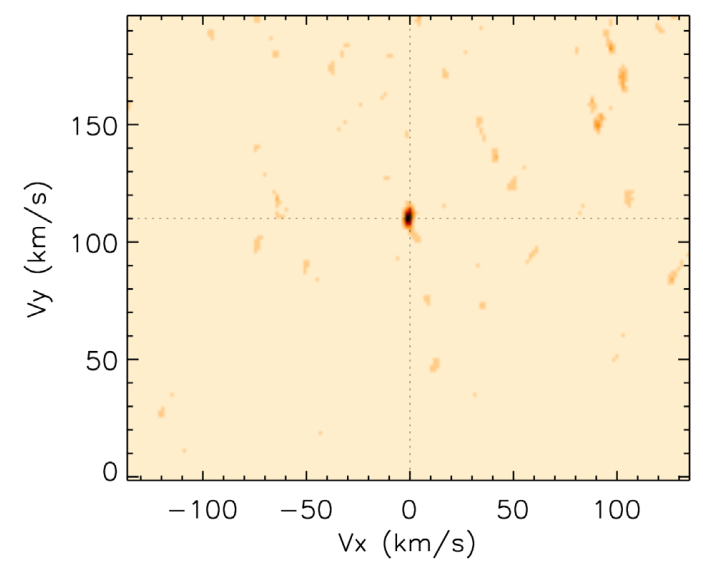

Figure 2. Doppler tomogram of the $\tau$ Boo b system using a CO linelist. The intersection of the dashed lines indicates the expected location of the planetary signature based on the detection of Brogi et al. (2012). CO is clearly detected in the Doppler tomogram at the expected location.

it is evident in the iterative process that Doppler tomography wants to push towards a lower $\chi^{2}$.

\section{DOPPLER TOMOGRAPHY COMPARISON TO CCF APPROACH}

In this section, we compare the ability of Doppler tomography to the conventional CCF analysis approach for the purposes of analysing time-series high-resolution Doppler observations of exoplanet atmospheres. This was done through tests using a series of simulated datasets. Each synthetic data set consists of 201 evenly spaced orbital phases from 0.25 to 0.75 . Lines were injected into the simulations assuming a systemic velocity $\left(v_{\text {sys }}\right)$ of $0 \mathrm{~km} \mathrm{~s}^{-1}$, a planetary radial velocity semi-amplitude $K_{\mathrm{p}}=150 \mathrm{~km} \mathrm{~s}^{-1}$, and a full width at half-maximum (FWHM) of $4.7 \mathrm{~km} \mathrm{~s}^{-1}$ unless otherwise stated in the relevant section. The wavelength coverage varies between each simulation (indicated where relevant), and is sampled by a pixel grid of $1 \mathrm{~km} \mathrm{~s}^{-1}$. Each simulation assumes white-noise (of varying levels between simulations), and the same noise map is applied to each data set. The Doppler tomography reconstructions were all run using a uniform default map.

We note that we have not considered the impact that temperature inversions can have on the line-shapes themselves within our linelist (e.g. Parmentier et al. 2018). This would require a full model atmosphere in order to produce the full complement of emission, absorption, and 'nulled' lines. This could be incorporated into Doppler tomography by using multiple line-lists capturing the main morphological line-shapes, and then fitting for these lines simultaneously (e.g. see Section 4.5).

In this paper we compare the Doppler tomograms (in terms of $v_{x}$ and $v_{y}$, as shown in Fig. 2) to the conventional way of showing the CCF analyses as a plot of the CCF strength as a function of $v_{\text {sys }}$ and $K_{\mathrm{p}}$ (e.g. see right-hand panel of Fig. 3). We note that, while $v_{y}$ and $K_{\mathrm{p}}$ are comparable on the $y$-axis of both the Doppler tomogram and CCF maps, $v_{x} \neq v_{\text {sys }}$. However, we maintain this format for presenting the CCF maps in this work as it allows for direct comparison to extrasolar planet atmosphere detections presented in the literature to date. We also note here that taking a cut along $v_{x}=0 \mathrm{~km} \mathrm{~s}^{-1}$ in a Doppler map and a cut at $v_{\text {sys }}=0 \mathrm{~km}$ $\mathrm{s}^{-1}$ in the CCFs (after removing the system's systemic velocity, as later presented in the first two columns of Fig. 6) are directly comparable.

In Appendix A we also compare a sample of the Doppler tomography results versus those obtained by back-projecting the CCFs in Fig. A1. This provides a direct comparison with Doppler tomography as it uses an identical $\mathrm{v}_{x}$ and $\mathrm{v}_{y}$ grid. We also present an alternative method of phase-folding the CCFs as a function of the orbital phase-offset and $K_{\mathrm{p}}$ (see Fig. A2).

\subsection{Noise properties}

Our first simulations investigated the relative performance of the techniques at different signal-to-noise levels. The signal-to-noise ratios reported in these simulations are per frame and are such that the values quoted represent the signal-to-noise of the planetary signal when combining all the lines present - as measured from the peak of the lines. However, we should note that since the lines are not confined to a single pixel (but span several), the actual signalto-noise ratio of the simulations will be higher (by a factor of 2-3 as estimated using the FWHM and pixel scale). For the tests described in this section, we have generated synthetic spectra using the same CO linelist as applied in our analysis of $\tau$ Boo data presented in Section 3.

Fig. 3 shows the results of the Doppler tomogram (left-hand panel) and the CCF analysis (right-hand panel), respectively, for a signal-to-noise ratio of $1 / 13$ (this $\mathrm{S} / \mathrm{N}$ was adopted as it gives
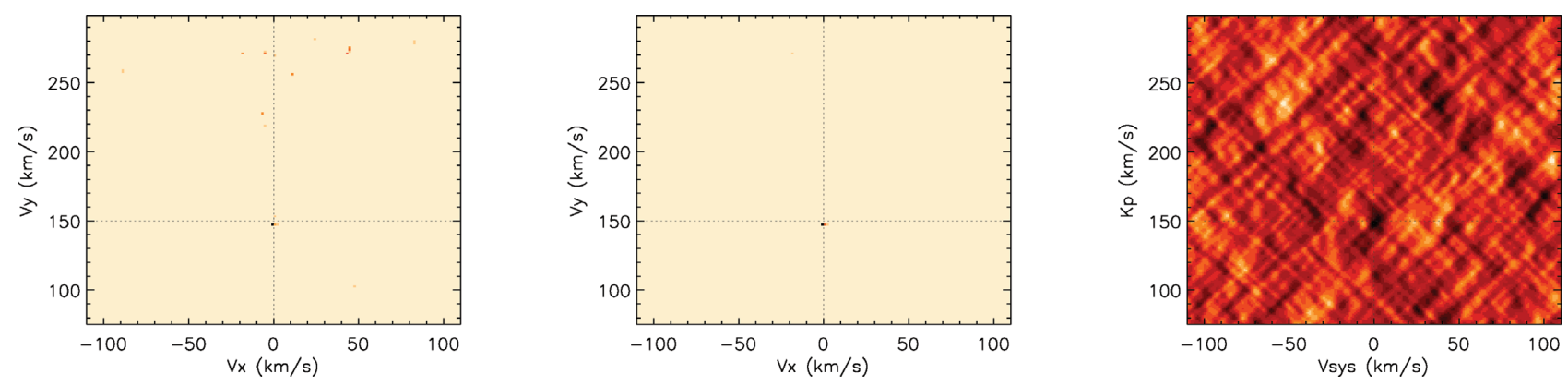

Figure 3. Left-hand panel: Doppler tomogram of the synthetic dataset as outlined in Section 4.1. The intersection of the dashed lines indicates the location of the injected planetary signal, which Doppler tomography recovers. Noise manifests itself as localized intensity spikes in the Doppler map and in this case is particularly evident at higher $v_{y}$ values. Central panel: The results of the signal coherency test whereby each pixel represents the minimum intensity value of the respective pixel in either the Doppler tomogram constructed using only odd-numbered spectra, or using only even-numbered spectra. Since random noise is not a truly coherent signal then most of the spikes are filtered out in this process, leaving only the true (injected) planet signal. Right-hand panel: The CCF map for the same dataset used in the Doppler tomography reconstruction shown on the left-hand panel. 

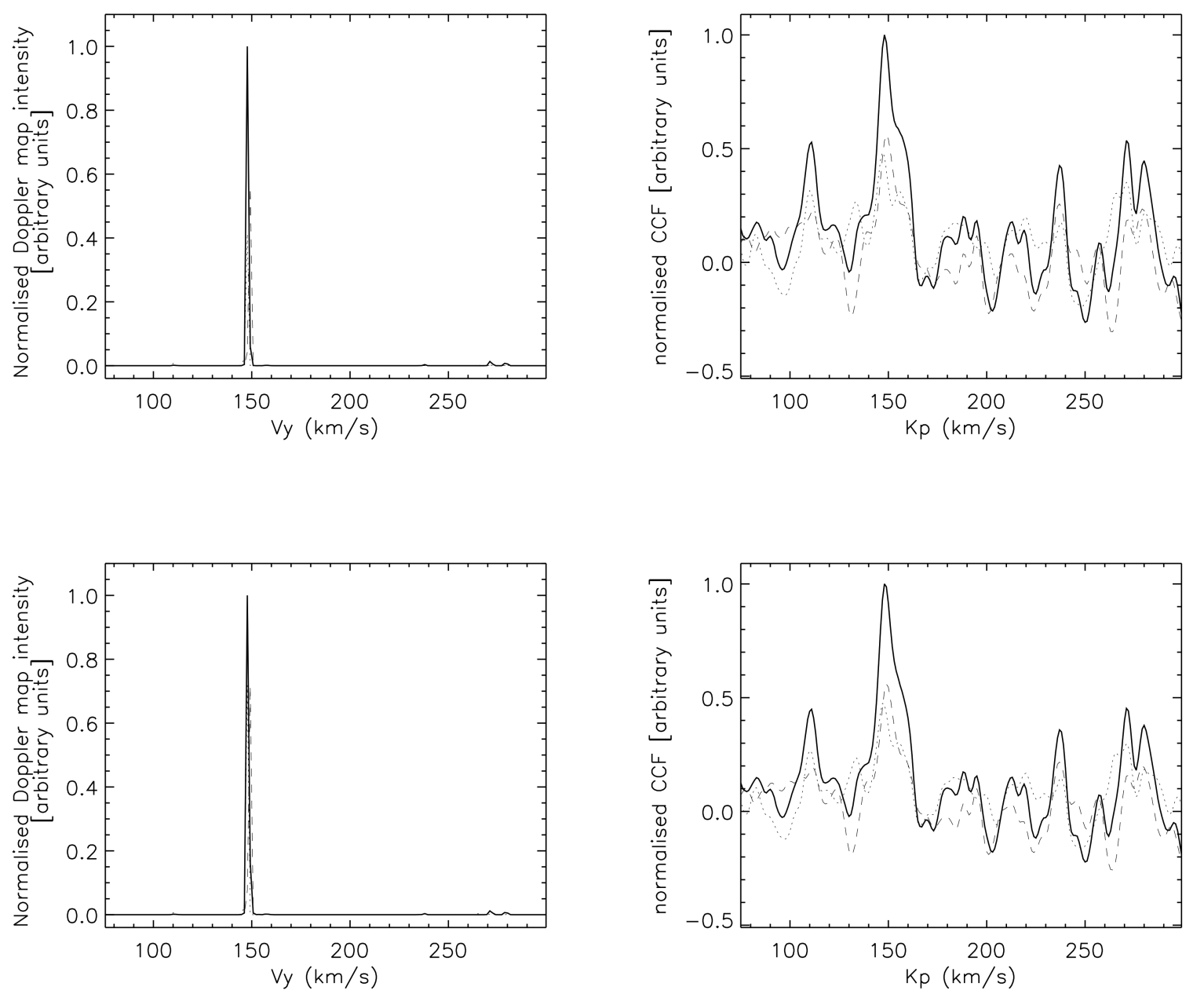

Figure 4. Left-hand panels: Cuts through the Doppler tomograms at $v_{x}=0 \mathrm{~km} \mathrm{~s}^{-1}$. Right-hand panel: Equivalent cut through the CCF maps at $v_{\mathrm{sys}}=0 \mathrm{~km}$ $\mathrm{s}^{-1}$. The top panels are for the maps shown in Fig. 3, while the bottom panels are for simulations with the same noise map but with a 1.44 times higher signal-to-noise ratio for comparison. Overplotted are the results when only even- (dashed lines) or odd-numbered (dotted lines) spectra are considered. The strengths of the odd/even tests are scaled relative to the signals generated by the full dataset results.

a realistically challenging signal to recover in the CCF analysis). The intersection of the dashed lines indicate the location of the injected planet. In all cases the images are plotted on a linear scale running from the minimum to the maximum value within the image. This scaling allows the noise properties within each map relative to the recovered line profile contributions to be visually compared.

While both the Doppler tomogram and CCF map recover the planetary signal, the first thing to note is the reduced noise levels in the Doppler tomograms. This is due to the fact that Doppler tomography fits the entire data set coupled to the use of the maximum entropy regularization statistic, which seeks to find the image of least information content. This has the effect of suppressing noise features that do not follow a radial velocity trajectory through the data as described by equation (1).

This can be further demonstrated in Fig. 4, which shows onedimensional cuts across regions of physical meaning in both the Doppler tomograms and the CCF maps. The top panel shows these cuts for the simulations used for Fig. 3, while the bottom panels show the same for a simulation where the signal-to-noise is a factor of 1.44 times higher. In the case of the Doppler tomogram, the only cut across the map of physical significance is along the $v_{x}=0$ line (vertical dashed line in the Doppler tomograms), where $v_{y}$ maps along the direction of the planet's radial velocity semi-amplitude, $K_{\mathrm{p}}$ (though see Section 4.4). For the CCF maps, we present a cut along the $K_{\mathrm{p}}$ axis, which provides a direct comparison between the two methods. Each plot is normalized by the maximum value of the corresponding map presented in Fig. 3, thus a value of 1 means that it is the strongest signal present in the entire map. As can be seen in Fig. 4, as well as the reduced noise, the Doppler tomogram provides a much sharper line recovery compared to the CCF, despite the fact that a uniform default map has been implemented (generally a Gaussian smoothed default map would be better at recovering point-like line contributions to the Doppler tomogram).

Naturally, it is important to establish the robustness of any potential planetary signal. For CCF analysis, the significance of any detection is often estimated by measuring the signal peak-toRMS ratio, where the RMS is measured from the 'background' noise in the CCF maps. Unfortunately, such an analysis cannot be performed for Doppler tomograms as the maximum-entropy regularization statistic suppresses noise, leading to a highly nonGaussian noise distribution in the resultant maps. As can be seen in the left-hand panel of Fig. 3, while the injected planetary signal is clearly recovered, noise in Doppler tomograms manifest themselves as localized spots while the majority of the tomogram is noise free due to the regularization statistic. These noise 'spikes' could easily 
be misidentified as a bona fide planet signal if they lie close to the $v_{x}=0 \mathrm{~km} \mathrm{~s}^{-1}$ line along which any planet should lie.

In order to test the veracity of the recovered signal we examined the coherency of features in the map by constructing two additional Doppler tomograms. These tomograms were formed using only the odd-numbered spectra for one and the even-numbered spectra for the other, effectively forming two independent datasets. Only coherent signals in both data sets will appear in both tomograms, while the noise spikes will (typically) appear at different locations in the two tomograms. This odd-even approach is applied as standard to assess the reality of features produced by Doppler-imaging techniques (e.g. Barnes et al. 2004; Watson, Dhillon \& Shabaz 2006; Watson et al. 2007; Xiang et al. 2015). By then constructing a Doppler map formed by selecting the minimum intensity from either the even- or odd-phased Doppler tomogram at each pixel position, we can examine what features remain constant.

The central panel of Fig. 3 displays the results of this coherency test and, in comparison to the left-hand panel of Fig. 3, shows that this is an effective means for filtering out incoherent (noise) features. Naturally, it is possible that random noise could produce a broadly coherent signal in the Doppler tomogram that would not filter out, but under such circumstances a CCF analysis would suffer from the same effect. To show that the odd/even peaks are coherent in the Doppler tomogram, these are overplotted in Fig. 4. In this figure we also show the same for the CCF analysis for comparison.

In summary, at the signal-to-noise levels in the simulations we have conducted, Doppler tomography gives clearer signal detections. There are a number of reasons why one might expect Doppler tomography, in principle, to perform better than cross-correlation approaches. For example, aliasing of signals is a problem inherent to $\mathrm{CCF}$ analyses (as explored later in this section). This projects additional structures in the resulting CCF maps, which may both confuse the identification of the true molecular signal and act to reduce its significance. Since Doppler tomography actually fits to the lines themselves, aliasing issues are not so prominent (as demonstrated later), and the use of a regularizing statistic also helps to suppress noise by minimizing the information content of the final Doppler map.

There are, however, a number of possible caveats that should be considered. One is that Doppler tomography has the additional complication that a target $\chi^{2}$ has to be selected, and this is somewhat subjective - there is no such issue for CCF analysis. In addition, the maximum entropy regularization enforces a strict positivity criterion to the data. This effectively removes any negative signals (i.e. removes any positive noise lying above the continuum when an absorption signal is being searched for). This comes with the potential expense that a poor continuum fit can result in a planetary signal being lost, while the CCF analysis will be robust against this. We should note, however, that the results from our $\tau$ Boo analysis (Section 3) indicates that this is not a particular issue in reality as implemented here. One should also note that the strict positivity criterion does not mean that absorption lines cannot be traced as this simply requires flipping the sign of the data set. In addition, it is possible to map both absorption and emission lines simultaneously by adopting a 'virtual' pixel in the Doppler map that contributes to all wavelengths. This could then be used to accommodate any (deliberate or otherwise) continuum offsets to ensure absorption lines obeyed the positivity criterion, and the use of such a 'virtual' pixel has previously been adopted in Roche tomography reconstructions (see Watson et al. 2003).

\subsection{Impact of contaminating lines}

Here we probe the relative performance of the techniques when there is a nearby, strong contaminating line with the same radial velocity variation as the line of interest. This also provides a simple demonstration of the impact of errors (e.g. missing lines, incorrect line positions) in linelists used for such work (see, for example, Hoeijmakers et al. 2015).

In the first two simulations, the synthetic data set consists of two equal strength lines separated by $50 \mathrm{~km} \mathrm{~s}^{-1}$. In the first instance (see Fig. 5, Images A1 and A2) the noise was set such that the RMS of the map was 3.98 times greater than the line depth, and in the second instance the RMS was 6.31 times greater than the line depth (see Fig. 5, Images B1 and B2). The results of the Doppler tomogram are shown in the left-hand panel, with the comparison of the CCF results shown in the right-hand panel. The intersection of the dashed lines indicate the location of the injected planet.

In Fig. 6 we show one-dimensional cuts across regions of physical meaning in both the Doppler tomograms and the CCF maps. These cuts are similar to those presented in Fig. 4, however for the CCF maps we also includes cuts along the line of constant $K_{\mathrm{p}}$ (i.e. at the velocity of the planet but at different systemic velocities, $v_{\text {sys }}$ ). Again, as before, each plot is normalized by the maximum value of the corresponding map presented in Fig. 5 .

In both signal-to-noise cases, the true planet signature is the strongest feature present in the Doppler tomogram. This is not the case for the CCF map, which shows the most power arising from the contaminating line not included in the linelist, seen as a strong feature shifted by $v_{\text {sys }}=+50 \mathrm{~km} \mathrm{~s}^{-1}$ from the actual planet position in Fig. 5 A2 and B2 and Fig. 6 A3 and B3. Indeed, this could potentially yield confusion as to the nature of the signal and/or systemic velocity. This is in contrast to the Doppler tomogram, which has efficiently filtered out the contaminating line. The explanation for this can be seen in the Image $\mathrm{C}$ rows of Figs 5 and 6, which is the same data set used for Image A, but with the contaminating line now offset by $20 \mathrm{~km} \mathrm{~s}^{-1}$. The Doppler tomogram now shows a semicircular arc-structure to the right of the true planet position. This arises due to the fact that Doppler tomography sees the contaminating line as having a nonzero systemic velocity and therefore, from equation (1), it is forced to map the contribution of the contaminating line across a range of $\mathrm{v}_{x}$ and $\mathrm{v}_{y}$ pixels on the map. This essentially dilutes the signal in the Doppler map by spreading it over a larger area in velocity space which is then suppressed by the maximum entropy regularization. Thus, Doppler tomography is less affected by missing lines in the linelist, or potential contamination from other lines or species (see Section 4.3 for a further example of this). Indeed, in this particular case, careful phase selection can prevent any power from contaminating lines leaking on to the $v_{x}=0 \mathrm{~km} \mathrm{~s}^{-1}$ line in the Doppler tomogram, along which any planet signal is expected to lie if the systemic velocity and orbital phases are known for the system.

In Appendix A we also present the back-projections of the CCFs in Fig. A1 (Images A and C). In this case, the CCFs also show a similar semicircular artefact due to the nearby contaminating line, but also exhibits much higher noise levels than the Doppler tomogram. We note that, in the case where the contaminating line source pattern is much more complicated than simply a nearby single line, Doppler tomography performs much better than the CCF back-projection method (see Sections 4.3 and 4.5) due to the fact that it fits all the data simultaneously. 


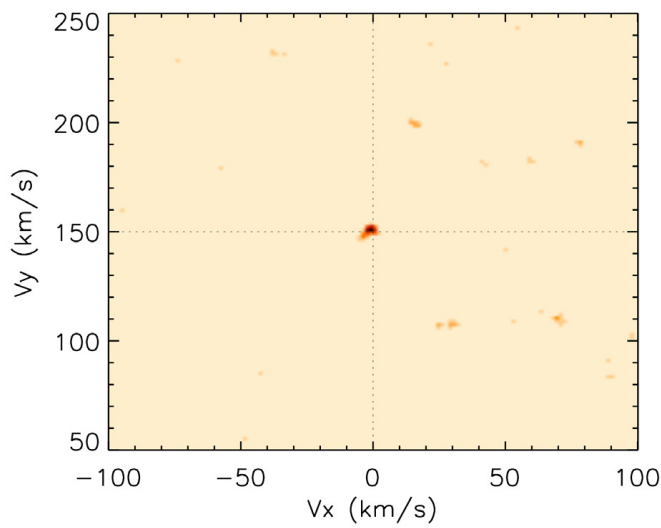

Image A1: Doppler tomogram, contaminating line at $+50 \mathrm{~km} \mathrm{~s}^{-1}$

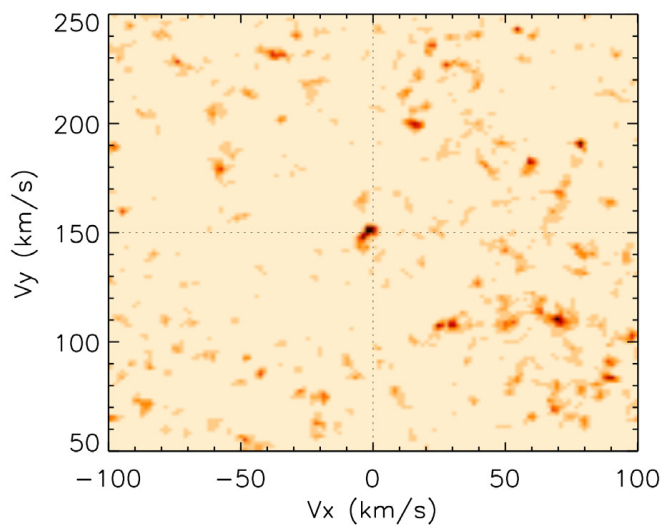

Image B1: As Image A1, but with a noise-level $~ 1.6$ times higher

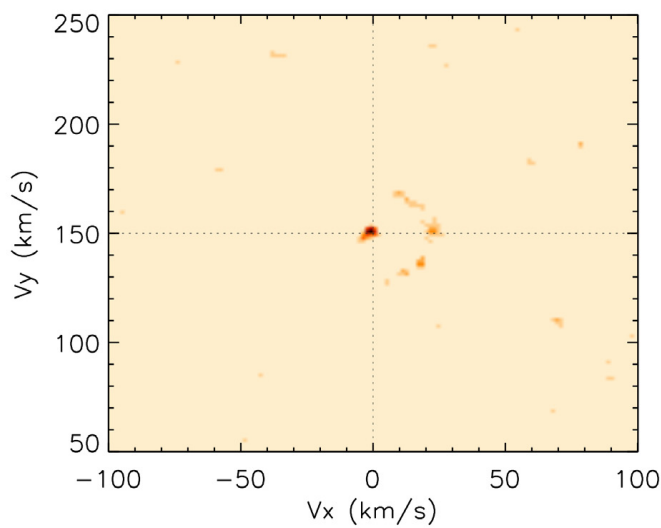

Image C1: Doppler tomogram, contaminating line at $+20 \mathrm{~km} \mathrm{~s}^{-1}$

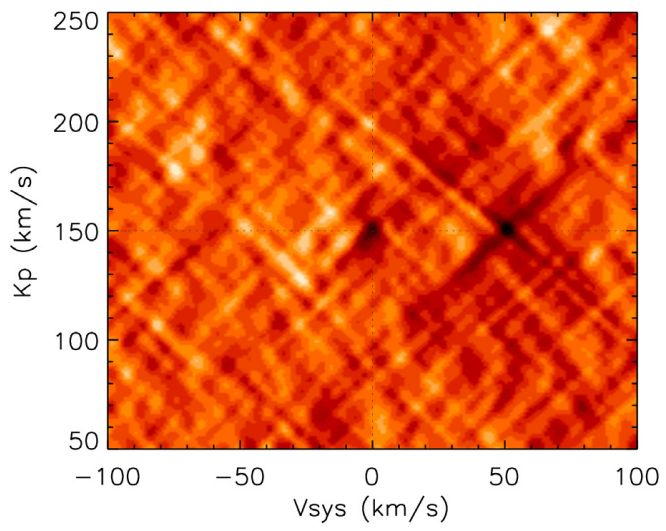

Image A2: CCF, contaminating line at $+50 \mathrm{~km} \mathrm{~s}^{-1}$

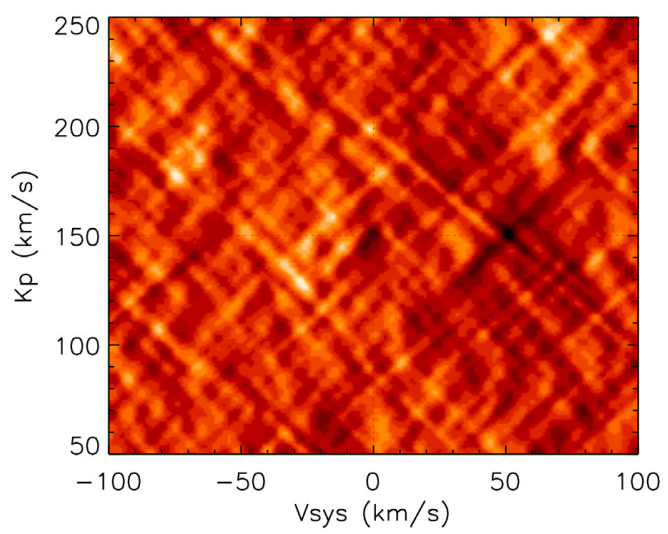

Image B2: As Image A2, but with a noise-level 1.6 times higher

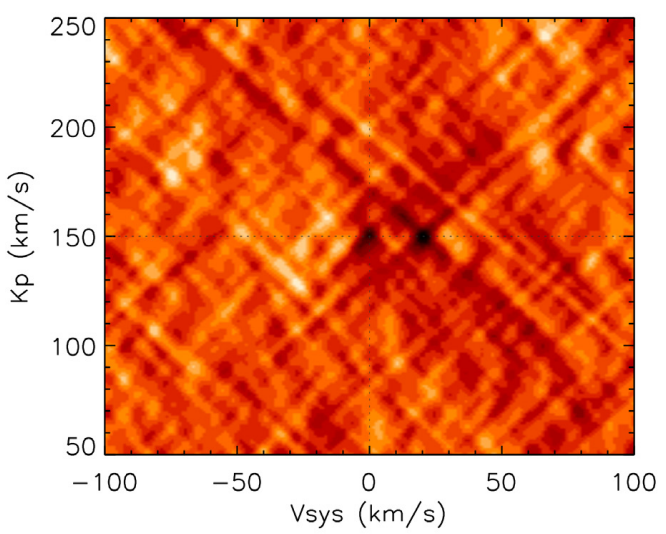

Image C2: CCF, contaminating line at $+20 \mathrm{~km} \mathrm{~s}^{-1}$

Figure 5. Comparison between Doppler tomography (left-hand panels) and the CCF method (right-hand panels) for different simulations. Images A and B show the effects for a contaminating line that is not included in the linelist, at $+50 \mathrm{~km} \mathrm{~s}^{-1}$. For Image A the noise level was set such that the RMS in the data was $\sim 4$ times the expected line strength, while in Image B the noise-level is set to 6.31 times the line strength. Image C shows a similar simulation, but now with the contaminating line offset by only $20 \mathrm{~km} \mathrm{~s}^{-1}$ and a noise level that is $\sim 4$ times that of the targeted line. All images are linearly scaled from the minimum to the maximum values in the respective map. The intersection of the dashed lines indicate the location of the true injected signal. Images $\mathrm{D}$ and $\mathrm{E}$ show the impact of contaminating lines when targeting specific isotopologues of $\mathrm{CO}$. The data for both maps contain ${ }^{12} \mathrm{C}^{16} \mathrm{O}$ and ${ }^{12} \mathrm{C}^{17} \mathrm{O}$ at equal strength. In Image $\mathrm{D}$ the data are analysed using only the ${ }^{12} \mathrm{C}^{17} \mathrm{O}$ linelist, while in Image $\mathrm{E}$ only a ${ }^{12} \mathrm{C}^{16} \mathrm{O}$ linelist is used. Image $\mathrm{F}$ shows a simulation containing both ${ }^{12} \mathrm{C}^{16} \mathrm{O}$ and ${ }^{12} \mathrm{C}^{17} \mathrm{O}$, but now the SNR is reduced significantly, and the abundance of ${ }^{12} \mathrm{C}^{16} \mathrm{O}$ is enhanced by a factor of 3 . The results here show an analysis targeting only the weaker ${ }^{12} \mathrm{C}^{17} \mathrm{O}$. Image $\mathrm{G}$ shows the impact of a spurious phase-offset of +0.03 from the actual orbital phase. Images $\mathrm{H}$ and I show a more extreme case of Images D and $\mathrm{E}$, where the strength of ${ }^{12} \mathrm{C}^{16} \mathrm{O}$ is enhanced by a factor of 100 compared to ${ }^{12} \mathrm{C}^{17} \mathrm{O}$. In the case of the Doppler tomogram maps, we search for ${ }^{12} \mathrm{C}^{16} \mathrm{O}$ and ${ }^{12} \mathrm{C}^{17} \mathrm{O}$ simultaneously, while for the CCFs we target each molecule separately. Image $\mathrm{H}$ show the results for ${ }^{12} \mathrm{C}^{16} \mathrm{O}$ and Image $\mathrm{I}$ for ${ }^{12} \mathrm{C}^{17} \mathrm{O}$. As can be seen Doppler tomography recovers both species, while the $\mathrm{CCF}$ only clearly detects ${ }^{12} \mathrm{C}^{16} \mathrm{O}$. 


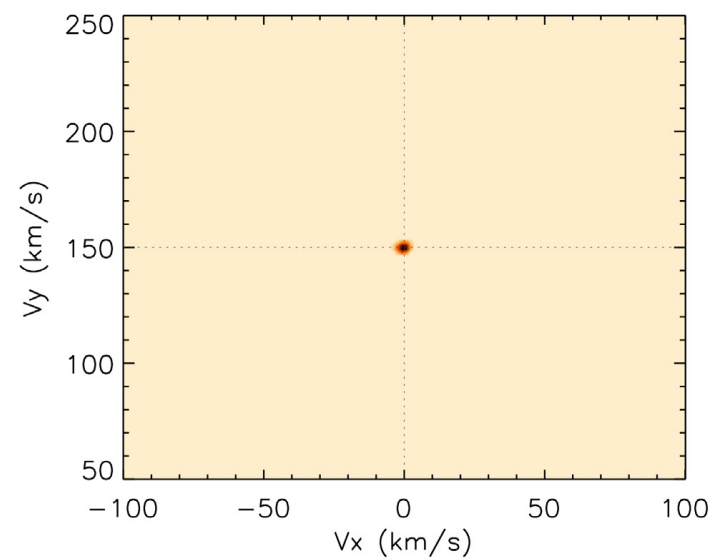

Image D1: Doppler tomogram for ${ }^{12} \mathrm{C}^{17} \mathrm{O}$

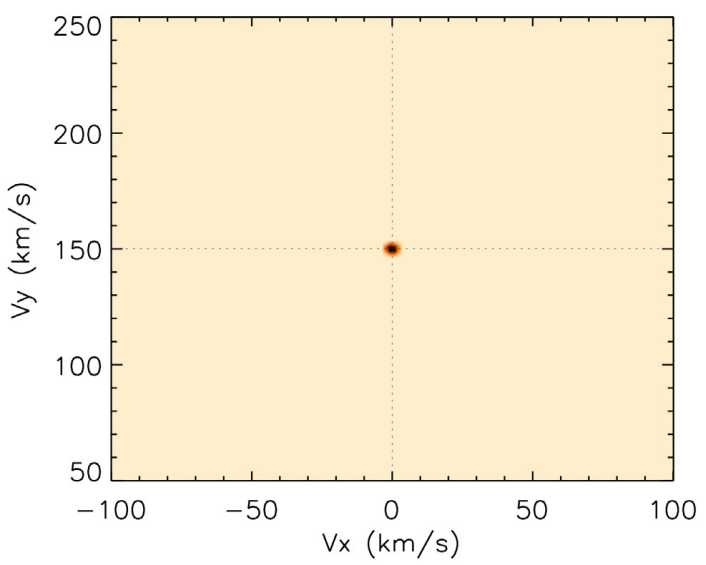

Image E1: Doppler tomogram for ${ }^{12} \mathrm{C}^{16} \mathrm{O}$

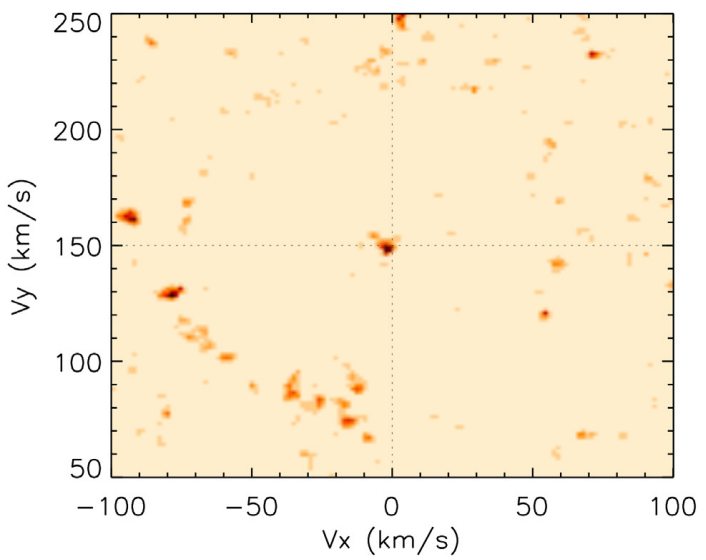

Image F1: Doppler tomogram for ${ }^{12} \mathrm{C}^{17} \mathrm{O}$

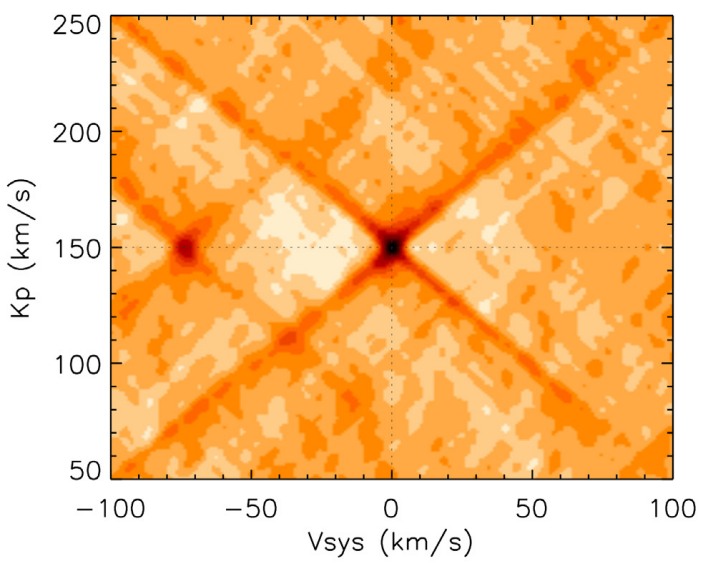

Image D2: CCF for ${ }^{12} \mathrm{C}^{17} \mathrm{O}$

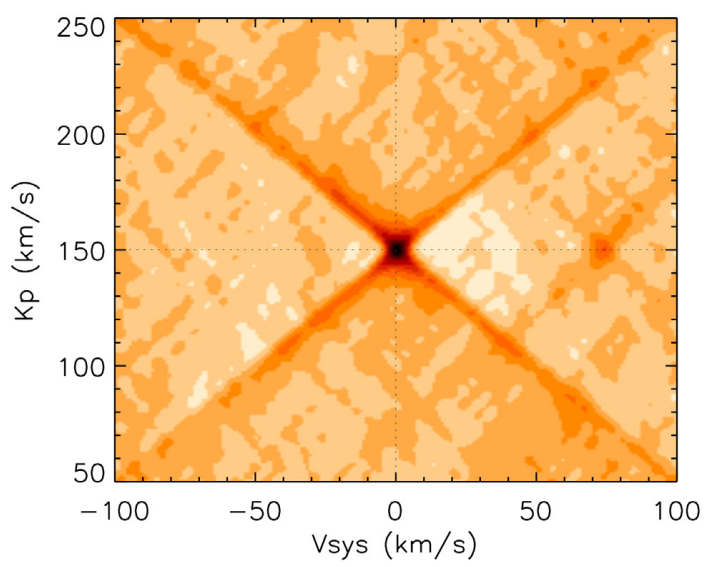

Image $\mathrm{E} 2$ : $\mathrm{CCF}$ for ${ }^{12} \mathrm{C}^{16} \mathrm{O}$

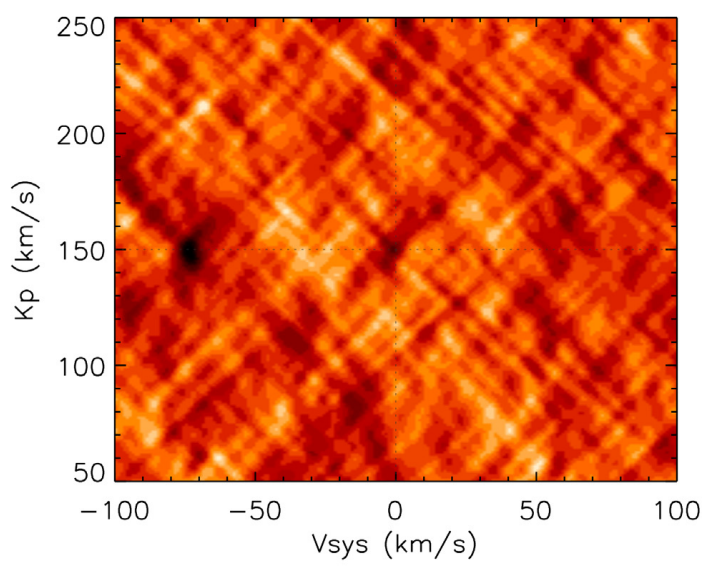

Image $\mathrm{F} 2$ : $\mathrm{CCF}$ for ${ }^{12} \mathrm{C}^{17} \mathrm{O}$

Figure 5 - continued

\subsection{Line-list confusion}

In our next set of simulations, we downloaded a $\mathrm{CO}$ linelist for both the ${ }^{12} \mathrm{C}^{16} \mathrm{O}$ and ${ }^{12} \mathrm{C}^{17} \mathrm{O}$ isotopologues obtained from the HITRAN database (Rothman et al. 2013) using HAPI (Kochanov et al. 2016). Both linelists were used to generate synthetic spectra covering a wavelength range from 22861.833 to $23503.069 \AA$ in $0.077 \AA$ steps. The ${ }^{12} \mathrm{C}^{16} \mathrm{O}$ contained 107 lines within this wavelength range, whereas the ${ }^{12} \mathrm{C}^{17} \mathrm{O}$ contained 53 lines. An unrealistic case of equal abundance of both isotopologues was assumed, and the signal-tonoise in each individual spectrum was set such that the resultant 


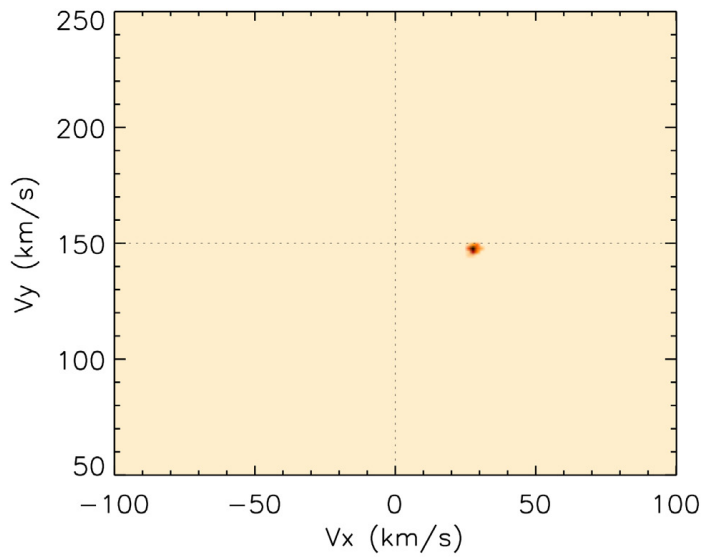

Image G1: Doppler tomogram for data with $\Delta \phi=+0.03$

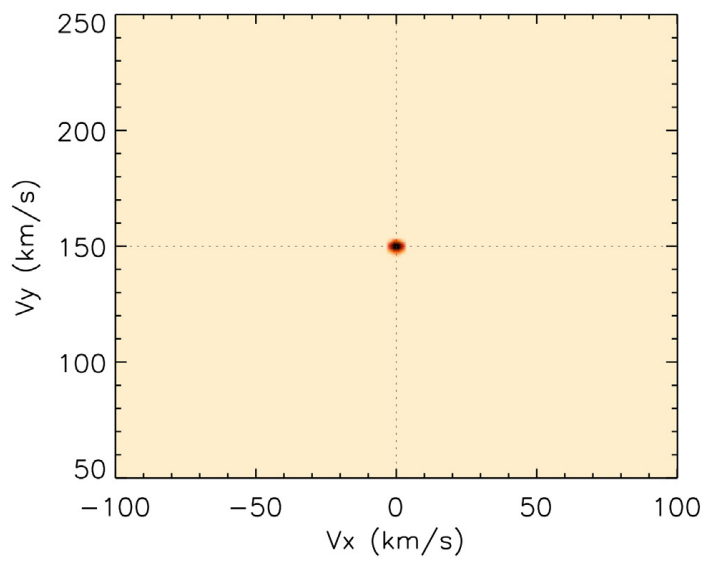

Image H1: Isotopologue test ${ }^{12} \mathrm{C}^{16} \mathrm{O}$ Doppler map

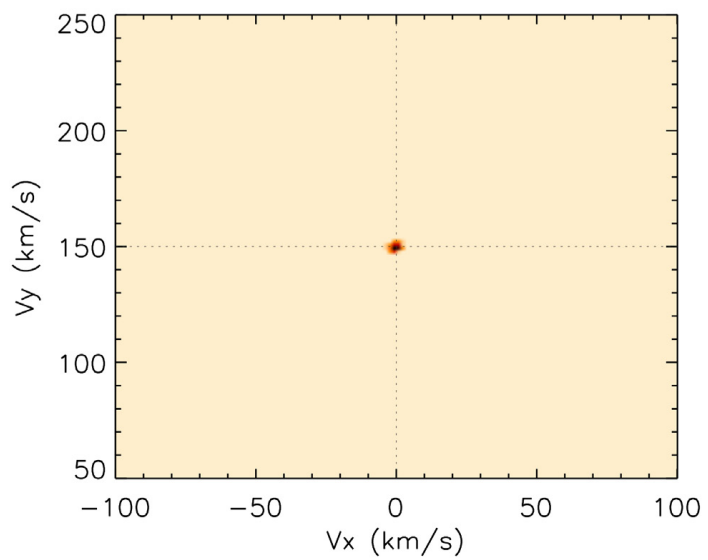

Image I1: Isotopologue test ${ }^{12} \mathrm{C}^{17} \mathrm{O}$ Doppler map

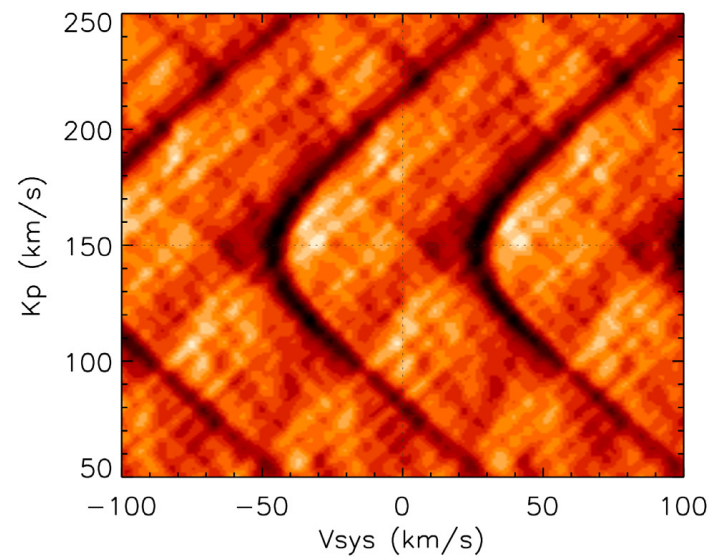

Image G2: CCF for data with $\Delta \phi=+0.03$

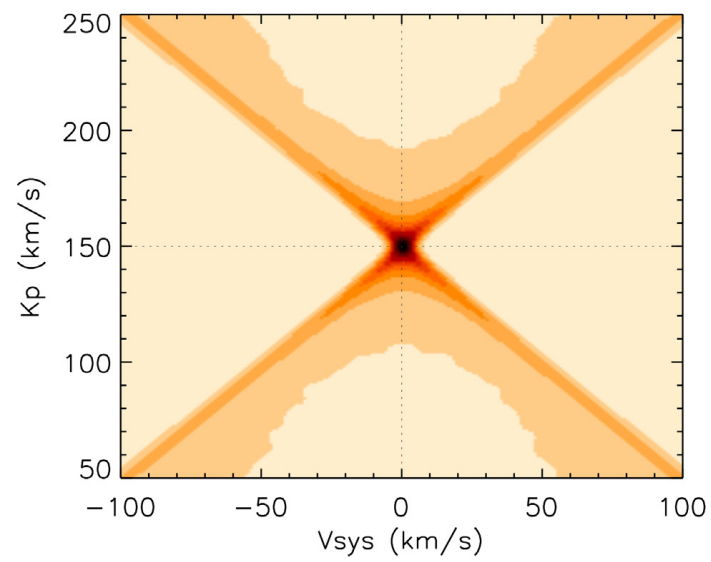

Image $\mathrm{H} 2$ : Isotopologue test ${ }^{12} \mathrm{C}^{16} \mathrm{O} \mathrm{CCF}$

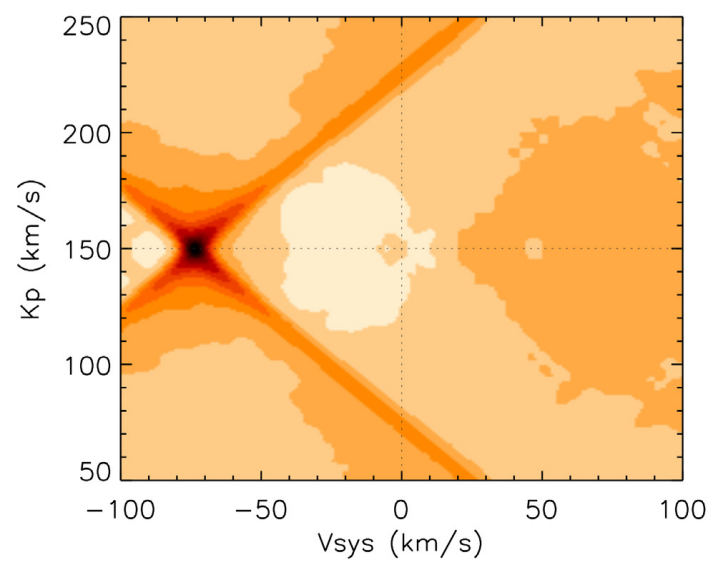

Image $\mathrm{I}$ : Isotopologue test ${ }^{12} \mathrm{C}^{17} \mathrm{O} \mathrm{CCF}$

Figure 5 - continued

CCF signal for ${ }^{12} \mathrm{C}^{17} \mathrm{O}$ in each individual spectrum approximately equalled the noise level (i.e. an approximately $1 \sigma$ detection of ${ }^{12} \mathrm{C}^{17} \mathrm{O}$ in one spectrum). Since ${ }^{12} \mathrm{C}^{16} \mathrm{O}$ has more lines in the wavelength range under consideration, it gives a stronger signal.
Both CCF maps and Doppler tomograms of the combined ${ }^{12} \mathrm{C}^{16} \mathrm{O}$ and ${ }^{12} \mathrm{C}^{17} \mathrm{O}$ synthetic spectra were reconstructed by first applying the ${ }^{12} \mathrm{C}^{17} \mathrm{O}$ linelist only (Fig. 5 Images D1 and D2), followed by applying the ${ }^{12} \mathrm{C}^{16} \mathrm{O}$ linelist only (Fig. 5 Images E1 and E2). The 


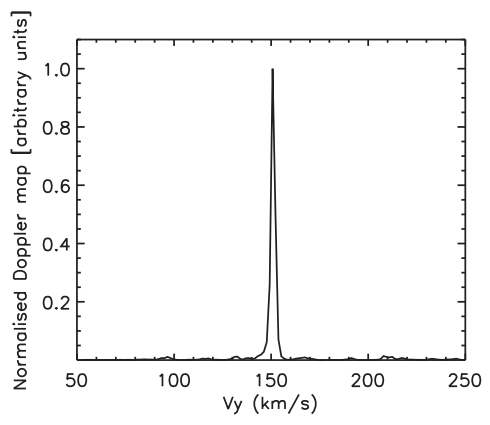

Image A1

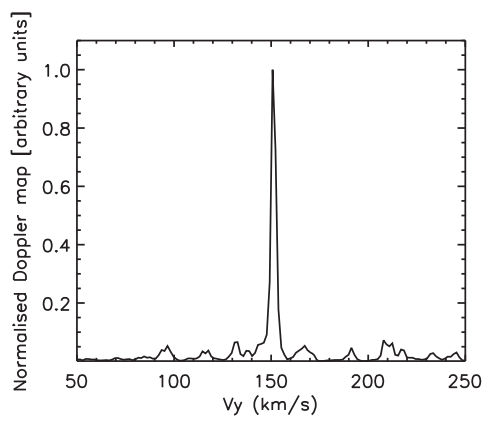

Image B1

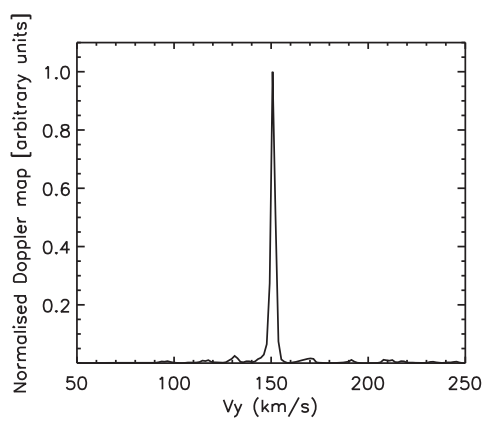

Image C1

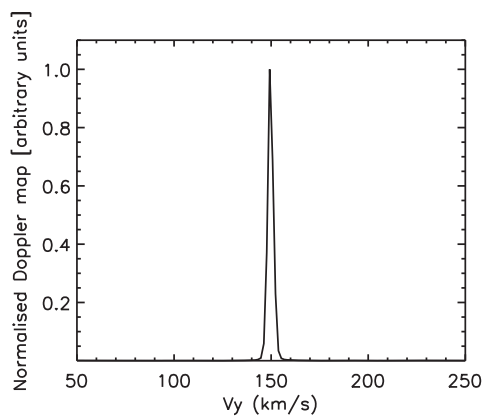

Image D1

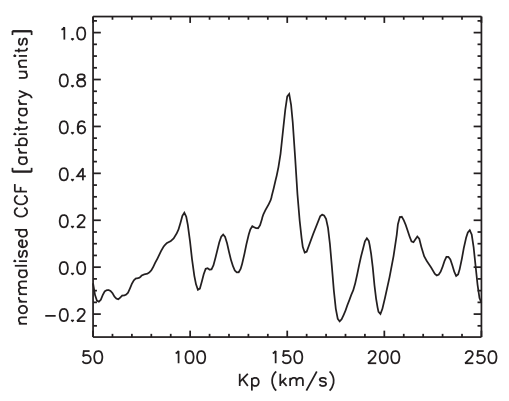

Image A2

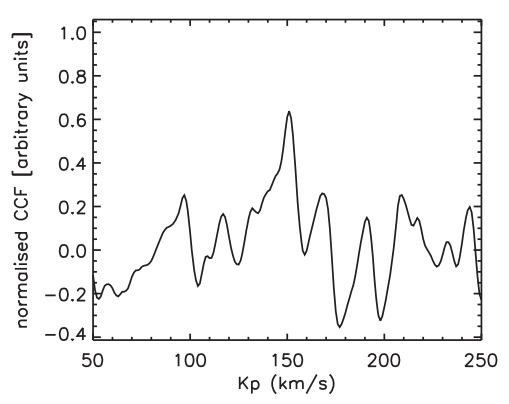

Image B2

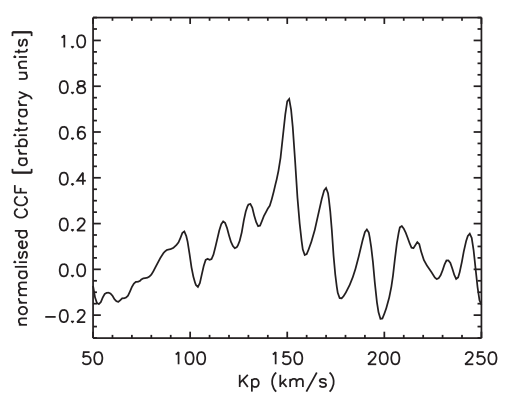

Image C2

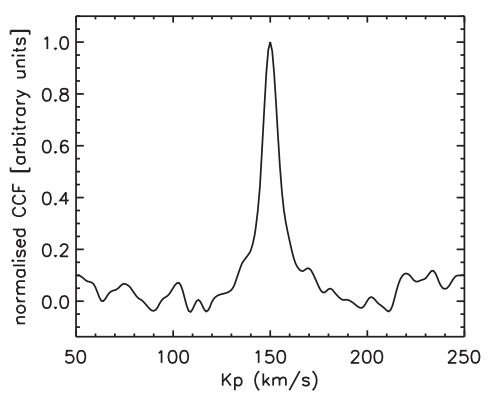

Image D2

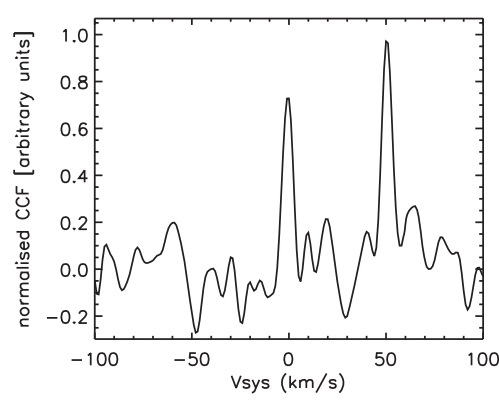

Image A3

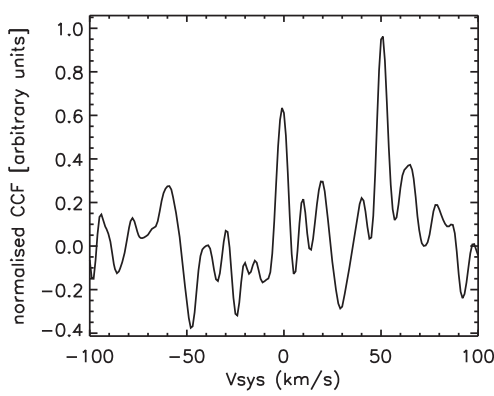

Image B3

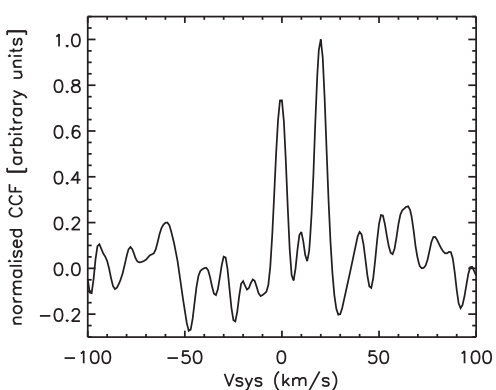

Image C3

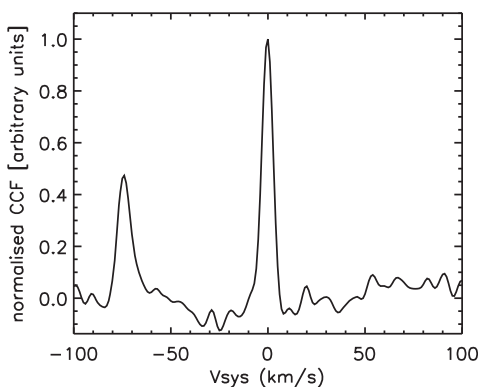

Image D3

Figure 6. Cuts through the Doppler tomograms and CCF maps from Fig. 5. The left-hand panels (column 1) show a cut through the Doppler tomograms at $v_{x}=0$ (where we expect the planet's signal if the phase is correct). For the CCFs we show cuts along both $K_{\mathrm{p}}$ for $v_{\mathrm{sys}}=0$ (middle panel, column 2 ) and along $v_{\text {sys }}$ at the injected planet velocity $K_{\mathrm{p}}=150 \mathrm{~km} \mathrm{~s}^{-1}$ (right-hand panels, column 3). The image identifier (A, B, C, etc.) correspond to those used in Fig. 5. Note that cuts across Image $\mathrm{G}$ are not shown, as the planet signal is no longer at $v_{x}=0$ in the Doppler tomogram and there is significant spurious structure in the CCF map. 


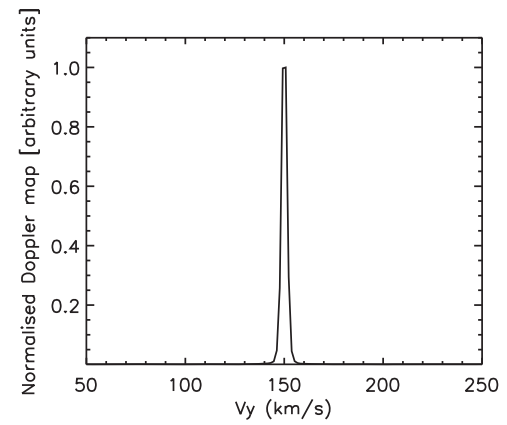

Image E1

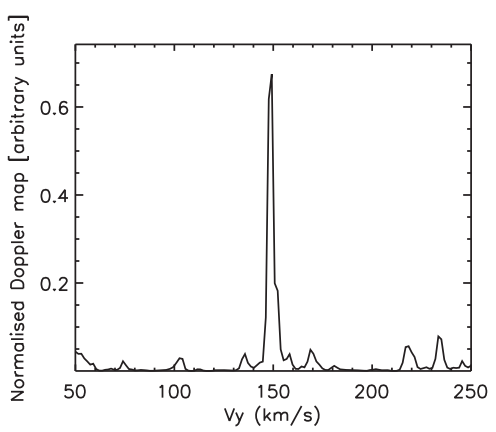

Image F1

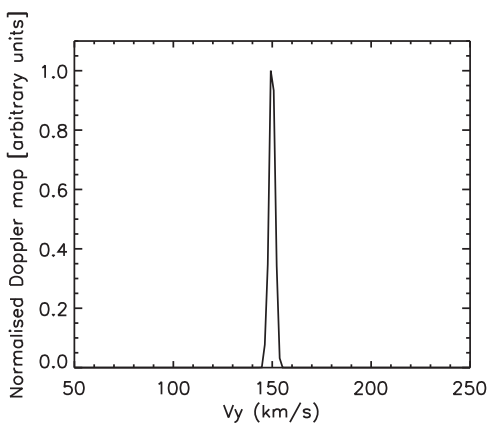

Image $\mathrm{H} 1$

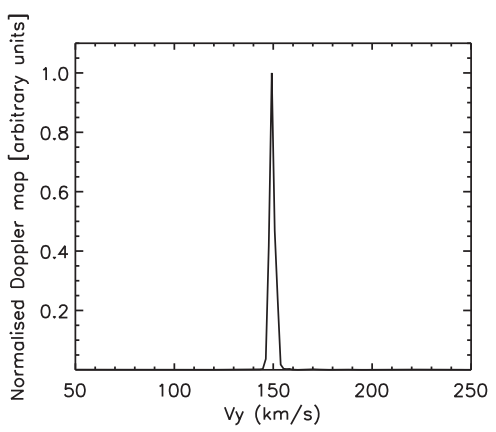

Image I1

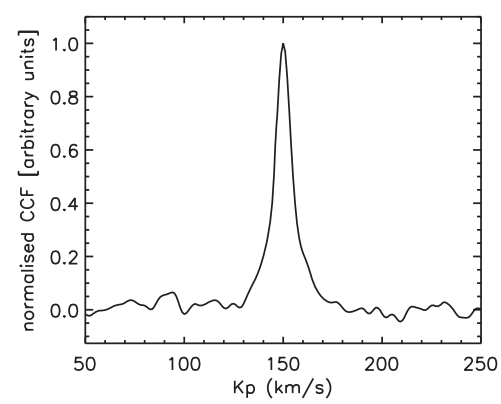

Image E2

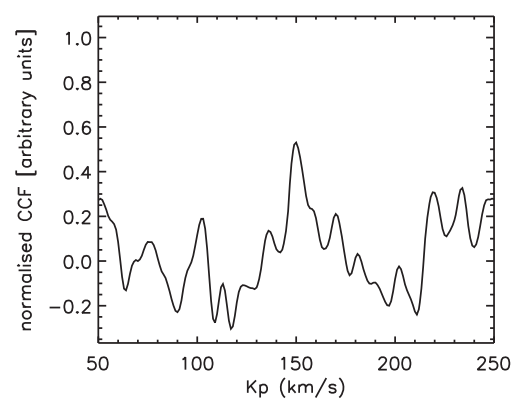

Image F2

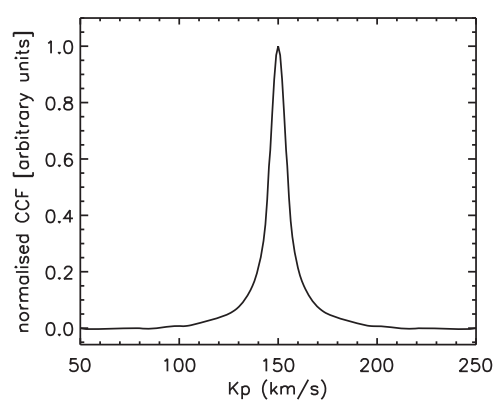

Image $\mathrm{H} 2$

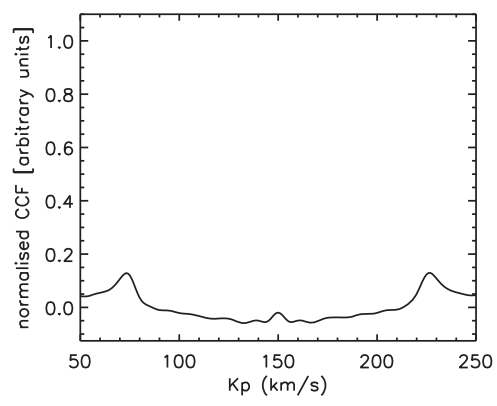

Image I2

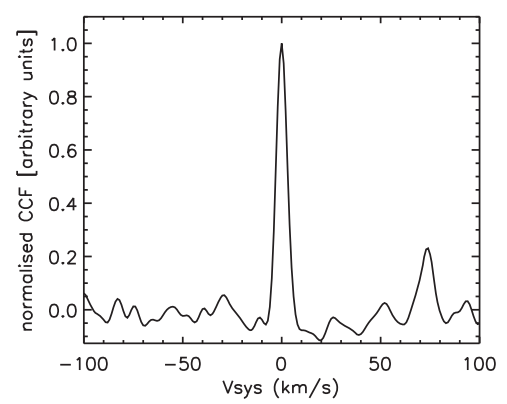

Image E3

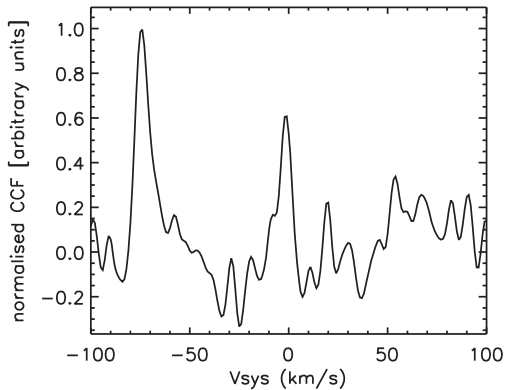

Image F3

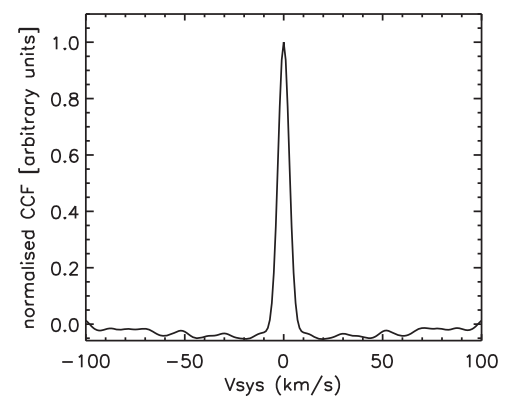

Image H3

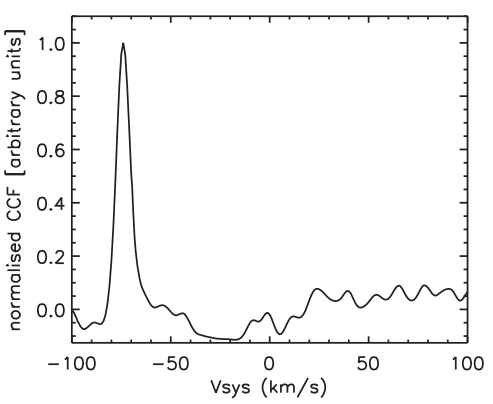

Image I3

Figure 6 - continued

respective cuts through these images are presented in panels D and $\mathrm{E}$ in Fig. 6.

While the planet signature is successfully recovered at the correct location in both the Doppler tomograms and the CCF maps, the CCF maps show strong evidence of a spurious signal at the correct $K_{\mathrm{p}}$, but with a systemic velocity of approximately $-75 \mathrm{~km} \mathrm{~s}^{-1}$ in Fig. 5 Image D2 (also see Fig. 6 Image D3) when the ${ }^{12} \mathrm{C}^{17} \mathrm{O}$ linelist is used. A similar, albeit weaker, signal is also seen at a systemic velocity of approximately $+75 \mathrm{~km} \mathrm{~s}^{-1}$ when the ${ }^{12} \mathrm{C}^{16} \mathrm{O}$ linelist is applied (Fig. 5 Image E2 and Fig. 6 Image E3). 
This is caused by cross-talk in the CCF process between the linelist being used, and the lines from the isotopologue present in the spectra that are not accounted for in the linelist. Since the ${ }^{12} \mathrm{C}^{16} \mathrm{O}$ isotopologue contains more lines, it shows up more prominently when the simulation is carried out using the ${ }^{12} \mathrm{C}^{17} \mathrm{O}$ linelist (Fig. 5 Image D2). While close isotopologues of the same molecular species tend to appear (very loosely) as shifted versions of one other, the typical wavelength shifts of the lines are of the order of 100 's $\mathrm{km} \mathrm{s}^{-1}$. Thus, it is not this overall 'shift' between the two isotopologues that results in the cross-talk. Rather, the cause is slightly more subtle. For molecules such as CO, the line pattern tends to repeat fairly regularly - thus even if there is a large wavelength shift between isotopologues, there is still a high possibility of a similar pattern of lines appearing near the lines of interest, and it is this that confuses the CCFs. It is clear from the Doppler tomograms, however, that there is no such confusion - as once more the erroneous lines are greatly smeared out in velocity space and suppressed by the regularization process as outlined in Section 4.3.

Again, in Appendix A, we present the back-projections of the CCFs in Fig. A1 (Image D). In this case, the CCF back-projection yields a semicircular artefact similar to those seen in the single contaminating line case presented in Section 4.3. As in that case, the CCF sees the nearly repeating line-pattern again, whereas Doppler tomography is able to suppress this erroneous signal.

We provide another example of this, where once more we have analysed the combined ${ }^{12} \mathrm{C}^{16} \mathrm{O}+{ }^{12} \mathrm{C}^{17} \mathrm{O}$ synthetic spectra assuming only the presence of the ${ }^{12} \mathrm{C}^{17} \mathrm{O}$ isotopologue. On this occasion the noise level with respect to the ${ }^{12} \mathrm{C}^{17} \mathrm{O}$ lines has been increased by a factor of 18 , and the line strengths of the ${ }^{12} \mathrm{C}^{16} \mathrm{O}$ isotopologue has been enhanced by a factor of 3 . The correct planet signature in the CCFs (Figs 5 and 6 Image F2) is now becoming difficult to discern, while it is still clearly picked up in the corresponding Doppler tomogram. We note that the peak in the Doppler tomogram signal at the planet location in Fig. 6 Image F1 is at 0.47 . In actual fact, the planet signature is still the strongest peak in the Doppler tomogram, but happens to land on a pixel adjacent to the correct location. Again, there is no sign of confusion due to cross-talk in the Doppler maps. The same cannot be said for the CCF map, which is dominated by the erroneous power injected into the signal by the presence of an unaccounted isotopologue. The ability of Doppler tomography to cleanly separate multiple molecular species/isotopologues is further examined in Section 4.5.

\subsection{Phase offsets}

The results of both CCF analyses and Doppler tomography are affected in different ways by adopting incorrect values for the systemic velocity, $\gamma$, and the orbital phasing. In the case of CCF analysis, the systemic velocity is one of the parameters that are determined, and therefore it is robust against errors in $\gamma$. Doppler tomography is, in theory, sensitive to errors in the systemic velocity. However, in practice, the systemic velocity of planetary systems is usually known to extremely high precision for planets studied with the radial velocity technique. Even a reflex Doppler wobble of $\sim 100 \mathrm{~m}$ $\mathrm{s}^{-1}$ induced on the host star by a hot-Jupiter is insufficient to inject enough uncertainty into $\gamma$ to have any discernable impact on the Doppler tomogram. In addition, it can be easily measured directly from the observed spectra in order to identify any instrumental velocity shifts or calibration errors that may have arisen.

The orbital phasing, on the other hand, may be uncertain in the case of non-transiting planets where phase slippage over many orbits can mount up over time to yield a substantial phase offset. In the case of Doppler tomography, a phase offset $(\Delta \phi)$ merely results in a rotation of the planet signature by an angle of $\Delta \phi$ radians from the origin, while otherwise completely preserving the signal strength. This is simulated using a synthetic data set comprising of 100 lines evenly spaced by $5.6 \AA$ starting at a wavelength of $22900 \AA$. In this case, an error in the phasing of the planetary orbit of $\Delta \phi=+0.03$ was injected, where the assumed phase $\phi_{\text {ass }}$ is related to the true phase $\phi_{\text {true }}$ by $\phi_{\text {ass }}=\phi_{\text {true }}+\Delta \phi$.

The resulting Doppler tomogram is shown in Image G1 of Fig. 5. As can be seen, the planet signal is preserved but rotated about the origin by $\Delta \phi \times 360^{\circ}=10.8^{\circ}$. The value of $\sqrt{\mathrm{v}_{x}^{2}+\mathrm{v}_{y}^{2}}$ is also preserved, leading to its new location in the velocity map of $v_{x}=$ $147 \mathrm{~km} \mathrm{~s}^{-1}, v_{y}=28.1 \mathrm{~km} \mathrm{~s}^{-1}$. Thus Doppler tomography behaves in an entirely predictable way and preserves the planetary signature and radial velocity amplitude of the planet - and can be used to determine the correct phase offset. The same simulated data set was then analysed using the CCF analysis, and is shown in Image G2 of Fig. 5. This marks an important difference between currently implemented CCF techniques, which are sensitive to phase offsets (though see Appendix A for an alternative method of analysing CCFs) that may not be well constrained in many non-transiting planetary systems compared to Doppler tomography that is more sensitive to errors in $\gamma$ (which is normally one of the best known system parameters). While trial phase offsets can be searched using the CCF technique, as Image G2 of Fig. 5 shows, even small phase offsets can destroy a planetary signal, and thus may result in an erroneous non-detection. This is particularly pertinent for the recovery of very weak signals, and we note that all Doppler spectroscopy studies of non-transiting planets with CRIRES have so far required phase-shift corrections to be applied.

We note that carrying out a back-projection of the CCFs can largely mitigate against the impact of a phase offset (see Fig. A1, Image $\mathrm{G}$ ), resulting in the same rotation of the signal about the origin as seen in Doppler maps. However, both the complete elimination of any aliasing (ringing) from the repeating linelist pattern and the superior noise reduction in the Doppler map are clearly evident.

\subsection{Separation of species and isotopologues}

The identification of different species and isotopologues in exoplanet atmospheres is an important goal. Mollière \& Snellen (2019) have discussed isotopologue detection using the CCF technique in some detail, and approach the problem by removing the strongest planetary contributions in an iterative fashion. One of the advantages of Doppler tomography over conventional CCF analyses is that it can simultaneously fit multiple different linelists of different species, and iteratively adjust the relative strengths between the different species. To demonstrate this, the same synthetic spectrum containing both ${ }^{12} \mathrm{C}^{16} \mathrm{O}$ and ${ }^{12} \mathrm{C}^{17} \mathrm{O}$ isotopologues was analysed, but on this occasion the strength of the ${ }^{12} \mathrm{C}^{16} \mathrm{O}$ lines was enhanced to be $\sim 100$ times stronger than those of the ${ }^{12} \mathrm{C}^{17} \mathrm{O}$ isotopologue. The signal-to-noise was adjusted such that the resultant signal for ${ }^{12} \mathrm{C}^{17} \mathrm{O}$ in each individual spectrum approximately equalled the noise level (i.e. an approximately $1 \sigma$ detection of ${ }^{12} \mathrm{C}^{17} \mathrm{O}$ in one spectrum).

Doppler tomography was run as before, except on this occasion it was supplied linelists for both isotopologues to fit simultaneously. At the start of the Doppler tomography reconstruction it was assumed that the strength of the lines from both isotopologues was roughly equal, but the relative weighting between the two linelists was allowed to vary. The results of this simultaneous dual-linelist 
fitting is shown in Fig. 5 Image $\mathrm{H} 1$ (for the ${ }^{12} \mathrm{C}^{16} \mathrm{O}$ map) and Image I1 (for the ${ }^{12} \mathrm{C}^{17} \mathrm{O}$ map). As can be clearly seen, Doppler tomography is able to cleanly disentangle the signals from both isotopologues at the correct velocity, despite a contrast of 2 orders of magnitude. Indeed, at this signal-to-noise there is no discernable cross-talk between the Doppler tomograms. In addition, the peak intensity in the two Doppler maps differ by a factor of 107 - close to the injected difference in weighting between the isotopologues. The robustness of this process can also be seen in Fig. 6 (Image H1 and I1). By comparison, running the $\mathrm{CCF}$ using each linelist in turn fails to detect the weaker ${ }^{12} \mathrm{C}^{17} \mathrm{O}$ isotopologue, and is dominated by the contribution from ${ }^{12} \mathrm{C}^{16} \mathrm{O}$ (see Fig. 5 Image $\mathrm{H} 2$ and I2). The same result is found if one back-projects the CCFs (see Fig. A1, Images $\mathrm{H}$ and $\mathbf{J}$ ).

\section{CONCLUSIONS}

In all of the simulations conducted, Doppler tomography has proven itself to yield either comparable or better results than the standard CCF approach, or the additional CCF back-projection approach presented in Appendix A. We have so far yet to create a scenario where Doppler tomography yields poorer results, except in cases where the systemic velocity of the system is poorly constrained (leading to a ringing pattern in the Doppler tomogram). However, the systemic velocity is one of the best constrained parameters of exoplanets with radial-velocity measurements, and can also be easily measured with the same data set used for the exoplanet atmosphere detection itself. The technique is robust against orbital phase offsets (and can be used to measure them), can handle contaminating lines, and of particular interest is its capability of simultaneously mapping different line species of unknown relative strengths. With instruments such as CRIRES+ soon to come online at the time of writing, this ability may prove immensely useful for measuring multiple molecular species across its wider wavelength coverage. By doing so, it may be possible to robustly determine the relative abundances of the major $\mathrm{C}$ - and O-bearing molecules such as $\mathrm{H}_{2} \mathrm{O}, \mathrm{CO}_{2}$, and $\mathrm{CH}_{4}$. This would be crucial for determining the planetary $\mathrm{C} / \mathrm{O}$ abundance ratio (Brogi et al. 2014), which has been cited as having a potentially critical influence on the properties of (and hence our understanding of) hot-hydrogendominated atmospheres (e.g. Madhusudhan 2012).

\section{FUTURE WORK}

The aim of this paper was mainly to demonstrate some of the advantages of applying Doppler tomography to the task of detecting exoplanet atmospheres using time-series high-resolution spectroscopy. There are, however, a number of extensions and further considerations that should be investigated, some of which are outlined below.

One is to develop robust methods for determining planetary atmosphere detection significances with Doppler tomography. Possible routes for doing this include the use of a bootstrapping approach in order to assess the impact of noise on the Doppler tomograms (as implemented in other tomographical image reconstructions, e.g. Watson \& Dhillon 2001, and also in Doppler tomography analysis of binary systems by Wang et al. 2017, 2018), or phase scrambling. In order to implement such methods, it may be necessary to reconstruct the Doppler tomograms to a target entropy value, rather than to a target $\chi^{2}$ as currently implemented. This comes about due to the fact that both approaches will change the noise properties slightly or, in the latter option, scrambling the phase order of the data would render any bona fide signal indiscernible by Doppler tomography. As a result, it would no longer be possible to fit as closely to those data points. Therefore, fitting to the same $\chi^{2}$ would force the algorithm to fit more closely to noise in the data, thereby altering the noise properties of the resulting Doppler tomogram. While this would lead to a systematic underestimation of the significance of any detected signals, in the worst case scenarios it may result in the rejection of a real feature. Iterating to a constant entropy value would largely preserve the noise properties of the Doppler map, thereby helping to reveal subtle signatures. Adoption of a more sophisticated default map or regularization statistic may also deliver tangible gains in sensitivity.

Finally, Doppler tomography can be adapted to account for any phase variation in the changing day/night aspect of the planet. Such Modulated Doppler Tomography codes have been applied to other objects, and Steeghs (2003) has demonstrated that such adaptations delivers improved fits. This also leads to the prospect of constraining planetary phase curves directly from time-series high-resolution spectroscopy.

\section{ACKNOWLEDGEMENTS}

CAW would like to acknowledge support from UK Science Technology and Facility Council grant ST/P000312/1. NPG gratefully acknowledges support from the Royal Society in the form of a University Research Fellowship. The authors would like to thank the referee, Prof Dr. Ignas Snellen, for very helpful comments and suggestions.

\section{REFERENCES}

Barnes J. R., Lister T., Hilditch R., Collier Cameron A., 2004, MNRAS, 348,1321

Birkby J. L., de Kok R. J., Brogi M., de Mooij E. J. W., Schwarz H., Albrecht S., Snellen I. A. G., 2013, MNRAS, 436, 35

Birkby J. L., de Kok R. J., Brogi M., Schwarz H., Snellen I. A. G., 2017, AJ, 153, 138

Brogi M., Snellen I. A. G., de Kok R. J., Albrecht S., Birkby J., de Mooij E. J. W., 2012, Nature, 486, 502

Brogi M., Snellen I. A. G., de Kok R. J., Albrecht S., Birkby J. L., de Mooij E. J. W., 2013, ApJ, 767, 27

Brogi M., de Kok R. J., Birkby J. L., Schwarz H., Snellen I. A. G., 2014, A\&A, 565, 124

Brogi M., de Kok R. J., Albrecht S., Snellen I. A. G., Birkby J. L., Schwarz H., 2016, ApJ, 817, 106

Brogi M., Giacobbe P., Guilluy G., de Kok R. J., Sozzetti A., Mancini L., Bonomo A. S., 2018, A\&A, 615, A16

Butler R. P. et al., 2006, ApJ, 646, 505

Collier Cameron A., Horne K., Penny A., James D., 1999, Nature, 402, 751

de Kok R. J., Brogi M., Snellen I. A. G., Birkby J., Albrecht S., de Mooij E. J. W., 2013, A\&A, 554, 82

Hoeijmakers H. J., de Kok R. J., Snellen I. A. G., Birkby J. L., Schwarz H., 2015, A\&A, 575, 20

Hoeijmakers H. J., Snellen I. A. G., van Terwisga S. E., 2018, A\&A, 610, 47

Kaeufl H. U. et al., 2004, in Moorwood A. F. M., Iye M., eds, Proc. SPIE Conf. Ser. Vol. 5492, Ground-based Instrumentation for Astronomy. SPIE, Bellingham, p. 1218

Kochanov R. V., Gordon I. E., Rothman L. S., Wcislo P., Hill C., Wilzewski J. S., 2016, J. Quant. Spectrosc. Radiat. Transfer, 177, 15

Leigh C., Collier Cameron A., Horne K., Penny A., James D., 2003a, MNRAS, 344, 1271

Leigh C., Collier Cameron A., Horne K., Penny A., James D., 2003b, MNRAS, 346, L16 
Lockwood A. C., Johnson J. A., Bender C. F., Carr J. S., Barman T., Richert A. J. W., Blake G. A., 2014, ApJ, 783, L29

Madhusudhan N., 2012, ApJ, 758, 36

Marsh T. R., 2001, in Boffin H., Steeghs D., eds, Astrotomography: Indirect Imaging Methods in Observational Astronomy. Springer-Verlag Lecture Notes in Physics, Berlin, p. 1

Marsh T. R., Horne K., 1988, MNRAS, 235, 269

Martins J. H. C. et al., 2015, A\&A, 576, 134

Mollière P., Snellen I. A. G., 2019, A\&A, 622, 139

Morales-Rueda L., 2004, Astron. Nachr., 325, 193

Nugroho S. K., Kawahara H., Masuda K., Hirano T., Kotani T., Tajitsu A., 2017, AJ, 154, 221

Parmentier V. et al., 2018, A\&A, 617, A110

Piskorz D. et al., 2016, ApJ, 832, 131

Piskorz D. et al., 2017, AJ, 154, 78

Richards M. T., 2004, Astron. Nachr., 325, 229

Rodler F., Lopez-Morales M., Ribas I., 2012, ApJ, 753, L25

Rothman L. S. et al., 2013, J. Quant. Spectrosc. Radiat. Transfer, 130, 4

Schwope A. D., Staude A., Vogel J., Schwarz R., 2004, Astron. Nachr., 325, 197

Snellen I. A. G., de Kok R. J., de Mooij E. J. W., Albrecht S., 2010, Nature, 465,1049

Steeghs D., 2003, MNRAS, 344, 448

Steeghs D., 2004, Astron. Nachr., 325, 185

Vrtílek S. D., Quaintrell H., Boroson B., Shields M., 2004, Astron. Nachr., 325, 209

Wang L., Steeghs D., Casares J., Charles P. A., Muñoz-Darias T., Marsh T. R., Hynes R. I., O’Brien K., 2017, MNRAS, 466, 2261
Wang L., Steeghs D., Galloway D. K., Marsh T., Casares J., 2018, MNRAS, 478, 5174

Watson C. A., Dhillon V. S., 2001, MNRAS, 326, 67

Watson C. A., Dhillon V. S., Rutten R. G. M., Schwope A. D., 2003, MNRAS, 341, 129

Watson C. A., Dhillon V. S., Shabaz T., 2006, MNRAS, 368, 637

Watson C. A., Steeghs D., Shabaz T., Dhillon V. S., 2007, MNRAS, 382, 1105

Xiang Y., Gu S., Cameron A. C., Barnes J. R., 2015, MNRAS, 447, 567

\section{APPENDIX A: ALTERNATIVE CCF REPRESENTATIONS}

In this paper we have compared the Doppler tomograms to the conventional phase-folded CCFs that have been presented in the literature. As pointed out by the referee, this is not entirely a one-toone comparison, and therefore we have investigated two additional ways of representing the $\mathrm{CCF}$ analysis: back-projection, and phasefolding the CCFs as a function of the orbital phase-offset and $K_{\mathrm{p}}$ (keeping $v_{\text {sys }}$ constant at the correct value). These are presented in Figs A1 and A2, respectively. The back-projection approach follows that outlined in Section 2 (see Fig. 1 for a schematic), where a time-series of CCFs are integrated along different radialvelocity curves according to equation (1), and these can be directly compared to the Doppler maps presented in the main body of this paper. 


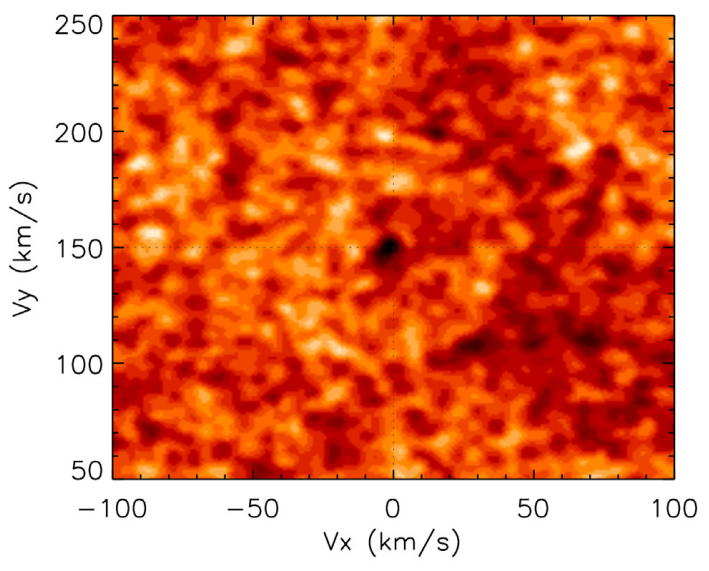

Image A: CCF back-projection, contaminating line at $+50 \mathrm{~km} \mathrm{~s}^{-1}$

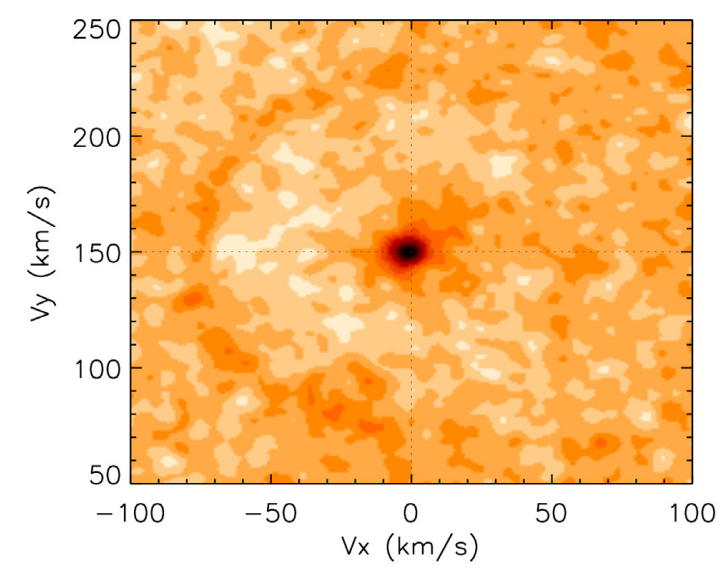

Image D: CCF back-projection for ${ }^{12} \mathrm{C}^{17} \mathrm{O}$.

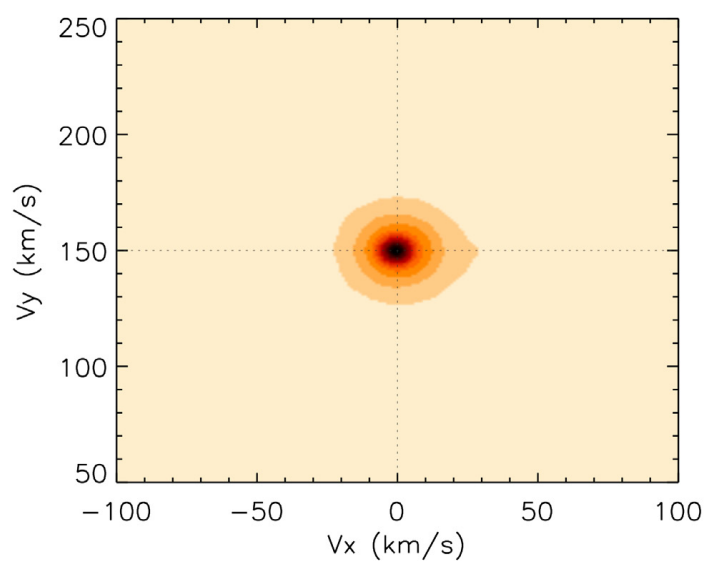

Image $\mathrm{H}$ : Isotopologue test ${ }^{12} \mathrm{C}^{16} \mathrm{O} \mathrm{CCF}$ back-projection

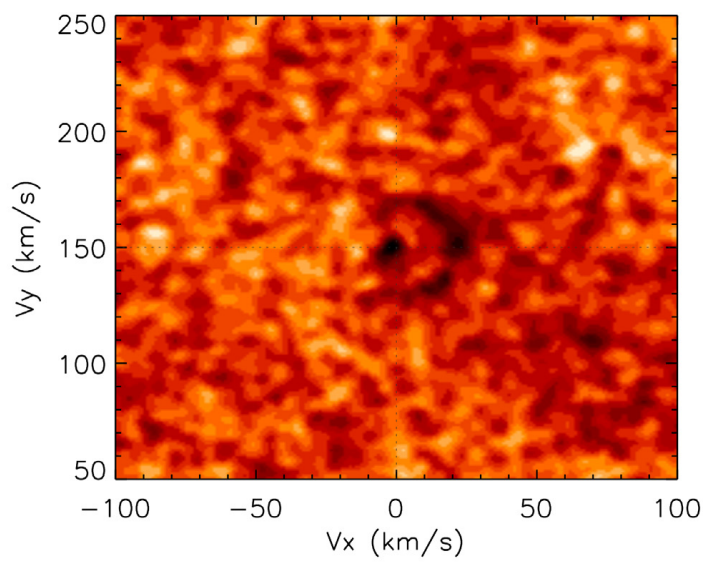

Image C: CCF back-projection, contaminating line at $+20 \mathrm{~km} \mathrm{~s}^{-1}$.

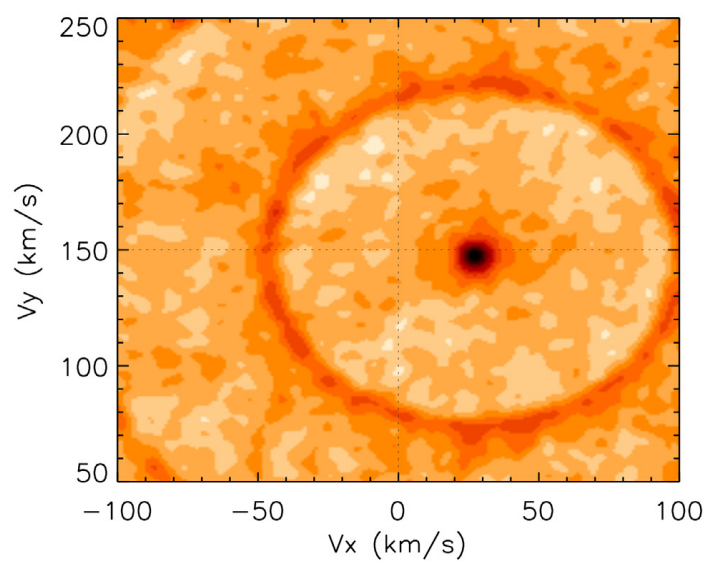

Image G: CCF back-projection for data with $\Delta \phi=+0.03$

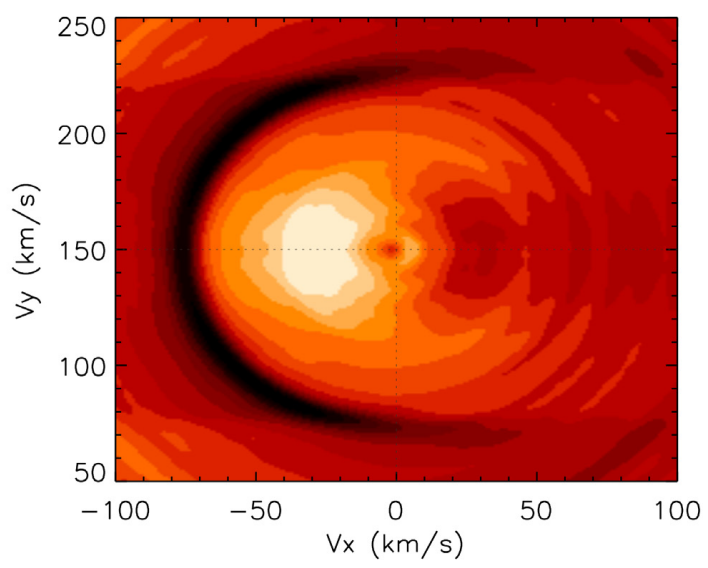

Image I: Isotopologue test ${ }^{12} \mathrm{C}^{17} \mathrm{O} \mathrm{CCF}$ back-projection

Figure A1. CCF back-projections for different simulations, the Image nomenclature mirrors that of the rest of the paper for ease of comparison. All images are linearly scaled from the minimum to the maximum values in the respective map. The intersection of the dashed lines indicate the location of the true injected signal. Images A and C show the effects of a contaminating line (that is not included in the linelist) offset from the line of interest by +50 and $+20 \mathrm{~km} \mathrm{~s}^{-1}$, respectively. Image D shows the case where data containing ${ }^{12} \mathrm{C}^{16} \mathrm{O}$ and ${ }^{12} \mathrm{C}^{17} \mathrm{O}$ at equal strength is analysed using only the ${ }^{12} \mathrm{C}^{17} \mathrm{O}$ linelist. Image $\mathrm{G}$ shows the impact of a spurious phase-offset of +0.03 from the actual orbital phase. Images $\mathrm{H}$ and I show the case where the strength of ${ }^{12} \mathrm{C}^{16} \mathrm{O}$ is enhanced by a factor of 100 compared to ${ }^{12} \mathrm{C}^{17} \mathrm{O}$. In the case of the Doppler tomogram maps presented in Fig. 5, we search for ${ }^{12} \mathrm{C}^{16} \mathrm{O}$ and ${ }^{12} \mathrm{C}^{17} \mathrm{O}$ simultaneously, while for the CCFs we target each molecule separately. Image $\mathrm{H}$ shows the results for ${ }^{12} \mathrm{C}^{16} \mathrm{O}$ and Image $\mathrm{I}$ for ${ }^{12} \mathrm{C}^{17} \mathrm{O}$. 


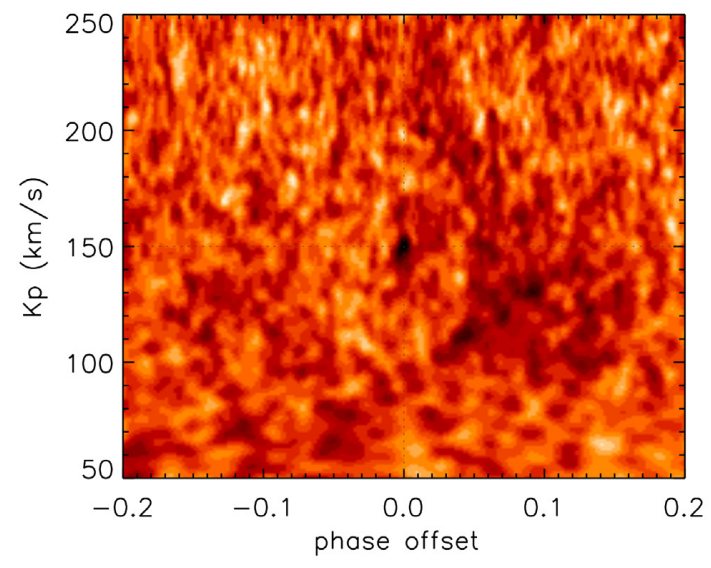

Image A: Phase-folded CCF, contaminating line at $+50 \mathrm{~km} \mathrm{~s}^{-1}$

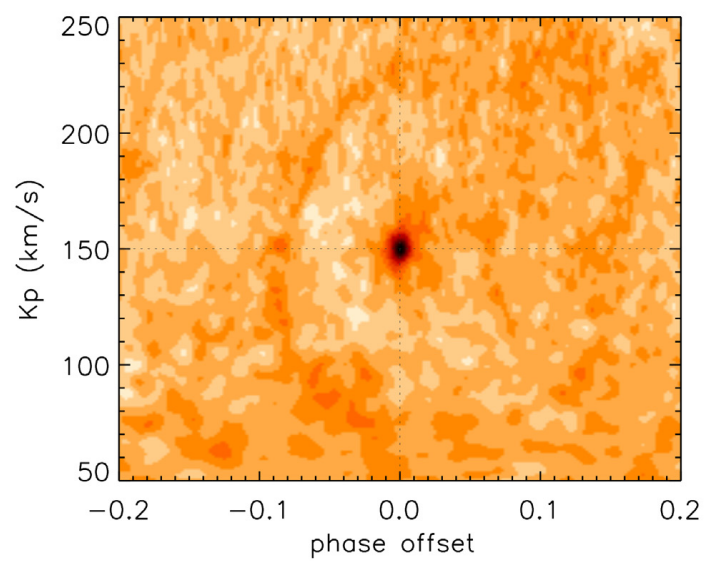

Image D: Phase-folded $\mathrm{CCF}$ for ${ }^{12} \mathrm{C}^{17} \mathrm{O}$.

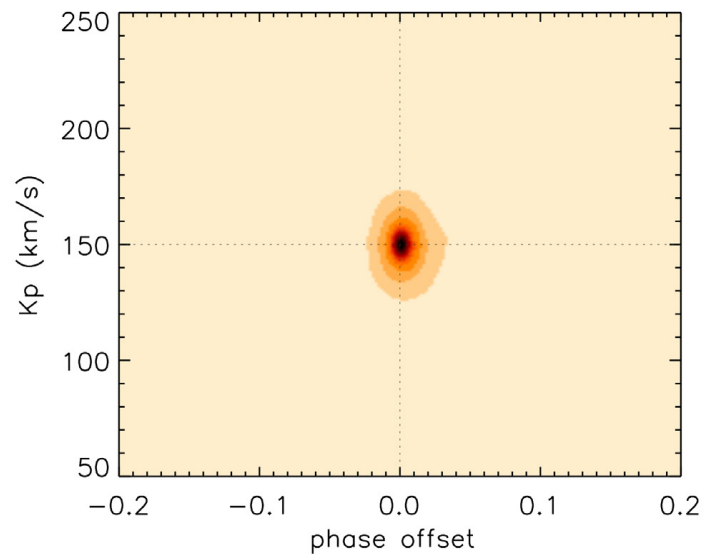

Image H: Phase-folded CCF, isotopologue test ${ }^{12} \mathrm{C}^{16} \mathrm{O}$

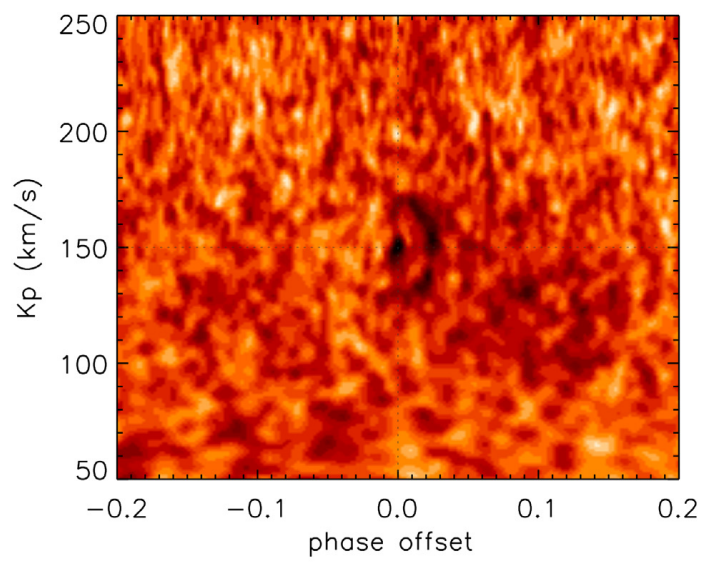

Image C: Phase-folded CCF, contaminating line at $+20 \mathrm{~km} \mathrm{~s}^{-1}$.

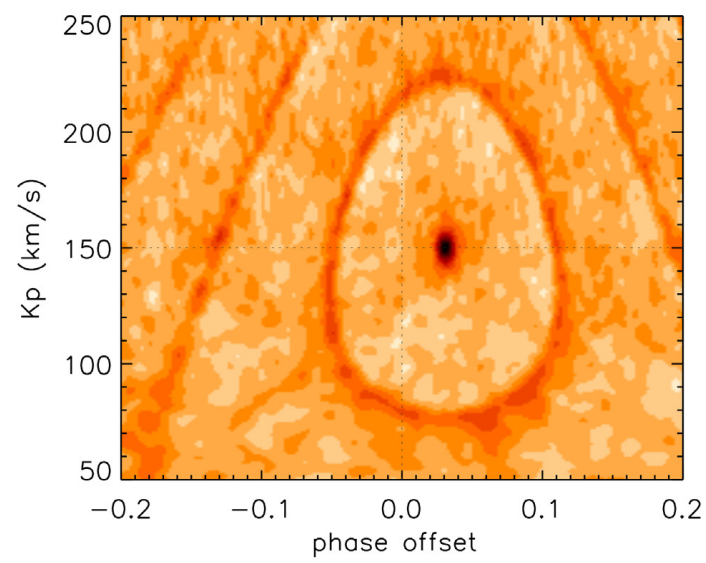

Image G: Phase-folded CCF for data with $\Delta \phi=+0.03$

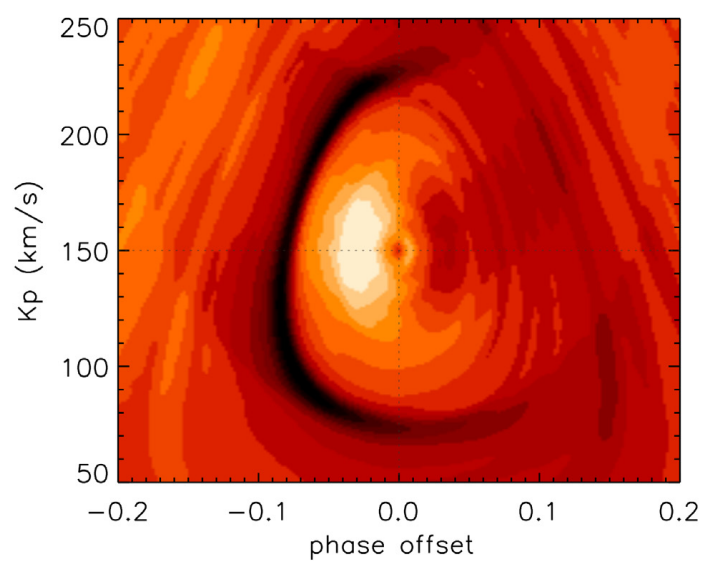

Image I: Phase-folded CCF, isotopologue test ${ }^{12} \mathrm{C}^{17} \mathrm{O}$

Figure A2. Alternative representation of the phase-folded CCFs for different simulations, in this case as a function of $K_{\mathrm{p}}$ and phase offset, keeping $v_{\text {sys }}$ fixed at the correct value. Again, the Image nomenclature mirrors that of the rest of the paper for ease of comparison.

This paper has been typeset from a $\mathrm{T}_{\mathrm{E}} \mathrm{X} / \mathrm{LT} \mathrm{E} \mathrm{X}$ file prepared by the author. 